PACIFIC JOURNAL OF MATHEMATICS

Vol. 181, No. 3, 1997

\title{
FINITE GROUPS ACTING ON HOMOLOGY MANIFOLDS
}

\author{
Michael Aschbacher
}

To the memory of Olga Taussky-Todd

\section{Introduction.}

In this paper we study homology manifolds $T$ admitting the action of a finite group preserving the structure of a regular $\mathrm{CW}$-complex on $T$. The $\mathrm{CW}$-complex is parameterized by a poset and the topological properties of the manifold are translated into a combinatorial setting via the poset. We concentrate on $n$-manifolds which admit a fairly rigid group of automorphisms transitive on the $n$-cells of the complex. This allows us to make yet another translation from a combinatorial into a group theoretic setting. We close by using our machinery to construct representations on manifolds of the Monster, the largest sporadic group. Some of these manifolds are of dimension 24, and hence candidates for examples to Hirzebruch's Prize Question in [HBJ], but unfortunately closer inspection shows the $\hat{A}$-genus of these manifolds is 0 rather than 1 , so none is a Hirzebruch manifold.

In order to state our results precisely we need to recall some definitions and introduce a few concepts. The geometric realization functor $T$ associates to each poset $X$ a topological space $T(X)$ and to each $x \in X$ a closed subspace $T(x)$ of $T(X)$. The cells $T(x), x \in X$, supply a cell structure on $T(X)$ parametrized by $X$. In addition there is a simplicial complex $\mathcal{O}(X)$ associated to $X$ called the order complex of $X$ and a canonical triangulation $\varphi: \mathcal{O}(X) \rightarrow T(X)$ of $T(X)$ by $\mathcal{O}(X)$.

An automorphism of a space $T$ with cell structure $(T(x): x \in X)$ is a homeomorphism $\alpha$ of $T$ permuting the cells and preserving the triangulation $\varphi: \mathcal{O}(X) \rightarrow T$. See Section 7 for a precise definition. In particular there is a natural isomorphism between the automorphisms of the space with cell structure and the automorphisms of $X$. The automorphism $\alpha$ is lower admissible if $\alpha$ induces the identity map on $T(x) \cap T(x) \alpha$ for each cell $T(x)$.

Assume $T$ is a connected homology $n$-manifold with cell structure supplied by a poset $X$. A reflection through an $(n-1)$-cell $T(x)$ is a nontrivial automorphism of the space $T$ with cell structure fixing each member of $T(x)$. There is at most one reflection through $T(x)$. 
Let $\mathcal{A}$ be a poset of height $n$ with a least element $a_{0}, I$ the set of elements of $\mathcal{A}$ of height $1, G$ a group, and $\rho: I \rightarrow G$ a map from $I$ into the set of involutions of $G$ such that $G=\langle\rho(I)\rangle$ is generated by the image of $\rho$. For $a \in \mathcal{A}$ let

$$
\mathcal{A}(\leq a)=\{b \in \mathcal{A}: b \leq a\}, \quad I(a)=I \cap \mathcal{A}(\leq a), \quad Q_{a}=\langle\rho(I(a))\rangle
$$

and $P(G, \rho, \mathcal{A})$ the poset with vertex set $\coprod_{a \in \mathcal{A}} G / Q_{a}$ and $Q_{a} g \leq Q_{b} h$ if and only if $a \leq b$ and $Q_{a} g \subseteq Q_{b} h$. The triple $(G, \rho, \mathcal{A})$ is thin if $\mathcal{A}\left(>a_{0}\right)$ is thin and residually connected. In the literature thin residually connected posets are sometimes called abstract polytopes; cf. [MS]. We show there is a bijection between the class of abstract polytopes $P$ admitting a lower admissible group of automorphisms transitive on maximal elements of $P$ and the class of thin triples; cf. Remark 1 in Section 4.

The triple $(G, \rho, \mathcal{A})$ is a manifold triple of height $n$ if $\mathcal{A}\left(>a_{0}\right)$ is a homology $(n-1)$-manifold and sphere, and for each $a \in \mathcal{A}$ of height at least 2, $P\left(Q_{a}, \rho_{a}, \mathcal{A}(<a)\right)$ is a homology sphere, where $\rho_{a}$ is the restriction of $\rho$ to $I(a)$. We prove the following two theorems which show that the notion of a connected homology manifold with cell structure admitting the rigid action of a group transitive on $n$-cells is equivalent to the notion of a manifold triple.

Theorem 1. Let $T$ be a connected homology n-manifold with cell structure supplied by a poset $X$, and $G$ a lower admissible group of automorphisms of $T$ transitive on $n$-cells of $T$. Let $T(x)$ be a $n$-cell of $T$. Then

(1) There is a reflection $\rho(i)$ through each $(n-1)$-cell $i$ below $x, G$ is generated by the set of all such reflections, and $G$ is the subgroup generated by all reflections of the automorphism group of the space $T$ with cell structure.

(2) Let $\mathcal{A}$ be the dual of $X(\leq x)$. Then $(G, \rho, \mathcal{A})$ is a manifold triple of rank $n$ and $X$ is isomorphic to the dual of $P(G, \rho, \mathcal{A})$.

Theorem 2. Let $(G, \rho, \mathcal{A})$ be a manifold triple of height $n$ and $X$ the dual of $P(G, \rho, \mathcal{A})$. Then the geometric realization $T=T(X)$ is a connected homology $n$ - manifold with cell structure supplied by $X$ and $G$ is a lower admissible group of automorphisms of the space $T$ transitive on the $n$-cells of $T$.

If $(G, \rho, \mathcal{A})$ is a manifold triple then for each $a \in \mathcal{A},\left(Q_{a}, \rho_{a}, \mathcal{A}(<a)\right)$ is also a manifold triple and $P_{a}=P\left(G_{a}, \rho_{a}, \mathcal{A}(<a)\right)$ is a homology sphere, so it is important to investigate the case where $P=P(G, \rho, \mathcal{A})$ is a homology sphere. One very strong result in this direction uses an idea of Davis in $[\mathbf{D}]$ 
and shows the isotropy groups $Q_{a}, a \in \mathcal{A}$, in our rigid action of $G$ on $T(P)$ are all spherical Coxeter groups:

Theorem 3. If $(G, \rho, \mathcal{A})$ is a manifold triple of height n such that $P(G, \rho, \mathcal{A})$ is a homology $n$-sphere then $(G, \rho(I))$ is a spherical Coxeter system.

It remains to decide the possible structures for the big cell $\mathcal{A}$ and the nature of the map $a \mapsto Q_{a}$ from $\mathcal{A}$ into the poset of all subgroups of $G$, particularly in the case where $P(G, \rho, \mathcal{A})$ is a homology sphere. Perhaps the following two results are our strongest in this direction.

Theorem 4. Let $(G, \rho, \mathcal{A})$ be a manifold triple of height $n$ such that $P=$ $P(G, \rho, \mathcal{A})$ is a homology sphere, $\rho$ is injective, and $I(a) \neq I(b)$ for $a<b$ in $\mathcal{A}$. Then $(G, \rho(I))$ is a spherical Coxeter system of rank $n+1$ or $n$ and $P$ is the Coxeter complex $C$ of $(G, \rho(I))$ or its suspension, (i.e. the join $C * S^{0}$ of $C$ with a 0 -sphere) respectively.

Theorem 5. Let $(G, \rho, \mathcal{A})$ be a thin triple such that $\mathcal{A}$ is of height $n$ with $\mathcal{A}\left(>a_{0}\right)$ a homology $(n-1)$-sphere and manifold, and for each $a \in \mathcal{A}$, $\left(Q_{a}, \rho(I(a))\right)$ is a spherical Coxeter system of rank the height of a. Then $(G, \rho, \mathcal{A})$ is a manifold triple.

Theorem 5 supplies one means for constructing representations of a group $G$ on manifolds. We use it in Section 13 to construct representations of the Monster of dimension ranging from 7 to 14, and observe in Example 2 in Section 12 that it can be used to construct representations on 2-dimensional manifolds of most any group generated by involutions. Then taking products of such examples we obtain many 24 dimensional representations of the Monster. See Section 11 for a brief discussion of Hirzebruch's Prize Question.

In Section 4 of $[\mathbf{D}]$, Davis proves results similar to Theorems 1 through 3 under the assumption that the big cell $\mathcal{A}\left(>a_{0}\right)$ is a simplex and $\rho$ is injective. Of course he does so in a different language. The preprint $[\mathbf{A}]$ contains results on manifolds parametrized by the cell complexes of [CCC], which are more general than regular $\mathrm{CW}$-complexes.

\section{Generalities on simplicial complexes and posets.}

Let $n$ be a nonnegative integer, $I=\{0, \ldots, n\}$, and $K$ an (abstract) $n$ dimensional simplicial complex. Write $K$ for the vertex set of $K$ and $\Sigma(K)$ for the set of simplices of $K$. Write $\Sigma^{k}(K)$ for the set of $k$-simplices of $K$. We call the $n$-simplices of $K$ chambers. A wall of a chamber $C$ is an $(n-1)$ simplex of $C$. The chamber graph of $K$ is the graph on the chambers of $K$ in which chambers $C$ and $D$ are adjacent if $C \cap D$ is a wall of $C$. 
We say $K$ is residually connected if $K$ is connected, $\operatorname{Link}_{K}(s)$ is connected for each $k$-simplex $s$ with $k<n-1$, and $\operatorname{Link}_{K}(s) \neq \varnothing$ for each $(n-1)$ simplex $s$.

A complex $K$ is a typed complex with type function $\tau$ if

$$
\tau: K \rightarrow I^{\prime}
$$

is a function from the vertex set of $K$ into some index set $I^{\prime}$ which is injective on simplices. For example if $I^{\prime}=I$ is of order $n+1$ this just says the graph of $K$ is a Tits geometry with type function $\tau$. The type of a simplex $s$ is its image $\tau(s) \subseteq I^{\prime}$.

A morphism $\alpha: K \rightarrow L$ of typed complexes over $I^{\prime}$ is a simplicial map preserving type. That is $\tau(v \alpha)=\tau(v)$ for each vertex $v$ of $K$. We write $\operatorname{Aut}(K, \tau)$ for the group of automorphisms of $K$ in the category of typed complexes.

A geometric complex is a typed complex over $I$ in which each simplex is contained in a chamber.

For example if $P$ is a poset, the order complex $\mathcal{O}(P)$ of $P$ is the simplicial complex with vertex set $P$ and simplices the finite chains of $P$. Define $P$ to be residually connected if $\mathcal{O}(P)$ is residually connected and define the chamber graph of $P$ to be that of $\mathcal{O}(P)$. If the set $P(\leq x)$ of vertices $y$ of $P$ with $y \leq x$ is of finite height then $\mathcal{O}(P)$ is a typed complex with type function the height function. The barycentric subdivision of a complex $K$ is the order complex of its poset of simplices, partially ordered by inclusion. Write $\operatorname{sd}(K)$ for the barycentric subdivision of $K$; thus $\operatorname{sd}(K)$ is a typed complex with respect to the height function.

An automorphism $g$ of $K$ is said to be admissible if whenever $g$ fixes a simplex $s$ of $K$ then $g$ fixes each vertex of $s$. Notice if $g$ is admissible then the set $\operatorname{Fix}(g)$ of all simplices of $K$ fixed by $g$ is a subcomplex of $K$.

Similarly $g$ is strongly admissible if $g$ fixes each vertex of $s \cap \mathrm{sg}$ for each simplex $s$ of $K$. A group $G$ of automorphisms is admissible, strongly admissible if each of its elements is admissible, strongly admissible, respectively. We say $G$ is topologically regular if whenever $g_{0}, \ldots, g_{k} \in G$ and $\left\{v_{0}, \ldots, v_{k}\right\}$ and $\left\{v_{0} g_{0}, \ldots, v_{k} g_{k}\right\}$ are $k$-simplices of $K$, then there exists $g \in G$ with $v_{i} g=v_{i} g_{i}$ for each $i$.

(2.1) Let $G$ be a group of automorphisms of $K$. Then

(1) $G$ is strongly admissible if and only if $v G \cap s=\{v\}$ for each simplex $s$ of $K$ and each $v \in s$.

(2) If $G$ is topologically regular then $G$ is strongly admissible.

(3) $G$ is topologically regular if and only if $G_{s}$ controls fusion in $\operatorname{Link}_{K}(s)$ for each simple $s$ of $K$. That is for each vertex $v$ of $K$, the pointwise 
stablizer $G_{s}$ of the simplex $s$ is transitive on $v G \cap \operatorname{Link}_{K}(s)$.

Proof. Parts (1) and (2) are easy and appear for example in Section 1 of Chapter III of $[\mathrm{Br}]$. Let $s=\left\{v_{0}, \ldots, v_{k}\right\}$ be a $k$-simplex of $K$. Assume $G$ is topologically regular and let $g \in G$ and $x, x g \in \operatorname{Link}_{K}(s)$. Then $s \cup\{x\}$ and $s \cup\{x g\}$ are simplices of $K$, so as $G$ is topologically regular there is $h \in G$ with $v_{i} h=v_{i}$ for each $i$ and $x h=x g$. That is $h \in G_{s}$ and $x h=x g$, so $G_{s}$ is transitive on $x G \cap \operatorname{Link}_{K}(s)$. Conversely assume $G_{s}$ controls fusion in $\operatorname{Link}_{K}(s)$ for each $s$. Let $\left\{v_{0} g_{0}, \ldots, v_{k} g_{k}\right\}$ be a simplex; we must show there is $g \in G$ with $v_{i} g=v_{i} g_{i}$ for each $i$. We proceed by induction on $k$; the remark is trivial when $k=0$. Let $t=s-\left\{v_{k}\right\}$; by induction on $k$ there is $h \in G$ with $v_{i} h=v_{i} g_{i}$ for $i \neq k$, so replacing $t$ by $t h$, we may assume $v_{i}=v_{i} g_{i}$ for $i \neq k$. Then as $G_{t}$ controls fusion in $\operatorname{Link}_{K}(t)$, there is $g \in G_{t}$ with $v_{k} g=v_{k} g_{k}$, completing the proof.

(2.2) Let $G$ be a group of automorphisms of $K$. Then

(1) $G$ is strongly admissible on $\operatorname{sd}(K)$.

(2) $G$ is topologically regular on $s d^{2}(K)$.

(3) If $G$ is admissible on $K$ then $\operatorname{Fix}_{s d(K)}(G)=s d\left(\operatorname{Fix}_{K}(G)\right)$ and $\operatorname{dim}(K)$ $=\operatorname{dim}(s d(K))$.

(4) $K$ and $s d(K)$ have the same homotopy type.

Proof. Part (4) is well known. Parts (1) and (2) are easy and appear as Proposition 1.1 in Chapter III of [Br]. Part (3) is trivial.

(2.3) Let $G$ be a group of automorphisms of $K$ and $C=\left\{v_{0}, \ldots, v_{n}\right\}$ a chamber of $K$. Then

(1) If there exists a $G$-invariant type function $\tau: K \rightarrow I^{\prime}$ making $K$ into a typed complex then $G$ is strongly admissible.

(2) If $G$ is strongly admissible then $G$ is admissible.

(3) If $G$ is strongly admissible, transitive on chambers, and each simplex of $K$ is contained in a chamber, then there is a unique type function $\tau$ : $K \rightarrow I$ preserved by $G$ with $\tau\left(v_{i}\right)=i$ and making $K$ into a geometric complex.

Proof. If $K$ is a typed complex with type function $\tau$ preserved by $G$, then for $g \in G$ and $s$ a simplex of $K, t=s \cap s g$ is the simplex of $s$ and of $s g$ of type $\tau(t)$, so as $g$ preserves type, $g$ fixes each vertex of $t$. That is $G$ is strongly admissible and (1) is established.

Part (2) is trivial. Assume $G$ is chamber transitive and each simplex is contained in a chamber. Then each vertex is in $v_{i} G$ for some $i$. Therefore if $G$ is strongly admissible, we can define $\tau: K \rightarrow I$ by $\tau\left(v_{i} g\right)=i$, and $\tau$ is 
well defined, since if $v_{i} g=v_{j}$ then $v_{j} \in C \cap C g$, so by strong admissiblity, $v_{i}=v_{i} g=v_{j}$. Visibly $\tau$ is the unique $G$-invariant function from $K$ to $I$ with $\tau\left(v_{i}\right)=i$. Finally if $s$ is a simplex of $K$ then $s g \subseteq C$ for some $g \in G$, so as $\tau$ is injective on $C$ and $G$-invariant, $\tau$ is injective on $s$. Thus $K$ is a geometric complex with type function $\tau$, completing the proof of (3).

(2.4) Let $G$ be a group of automorphisms of $K$ and $C=\left\{v_{0}, \ldots, v_{n}\right\}$ a chamber of $K$. Assume $G$ is admissible and chamber transitive and each simplex of $K$ is contained in a chamber. Then the following are equivalent:

(1) $G$ is strongly admissible.

(2) $G_{v_{i}}$ is chamber transitive on $\operatorname{Link}_{K}\left(v_{i}\right)$ for each $i \in I$.

(3) $G_{s}$ is chamber transitive on $\operatorname{Link}_{K}(s)$ for each simplex $s$ of $K$.

(4) $G$ is topologically regular on $K$.

Proof. Assume $G_{v_{i}}$ is chamber transitive on $\operatorname{Link}_{K}\left(v_{i}\right)$ for each $i \in I$. Suppose $v \in s \cap s g$ for some $g \in G$, some vertex $v$, and some simplex $s$. As $G$ is chamber transitive and $s$ is contained in a chamber, we may take $v=v_{i}$ and $s=C$. Then as $G_{v}$ is chamber transitive on $\operatorname{Link}_{K}(v)$, there is $h \in G_{v}$ with $C g h=C$. Now as $G$ is admissible, $g h$ fixes each vertex of $C$, so $v=v g h$ and hence $v g=v h^{-1}=v$. That is $G$ is strongly admissible. Thus (2) implies (1). On the other hand if $G$ is strongly admissible, $s \subseteq C$, and $d$ is a

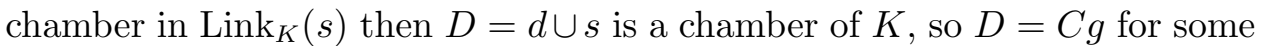
$g \in G$. Then $s \subseteq C \cap C g$, so as $G$ is strongly admissible, $g \in G_{s}$. That is (1) implies (3). Trivally, (3) impies (2). As $G$ is chamber transitive and each simplex is contained in a chamber, $(3)$ is equivalent to $G_{s}$ controlling fusion in $\operatorname{Link}_{K}(s)$, which is in turn equivalent to (4) by 2.1.3.

\section{Upper admissible representations.}

In this section $P$ is a poset of finite height $n$. A $k$-cell of $P$ is an element of $P$ of height $k$.

A group $G$ of automorphisms of $P$ is said to be lower admissible if each $g \in G$ fixes $P(\leq x) \cap P(\leq x g)$ pointwise for each $x \in P$. We define upper admissiblity dually.

Examples. (1) If $G$ is a strongly admissible group of automorphisms of the simplicial complex $K$ then $G$ is a lower admissible group of automorphisms of the poset $s d(K)$.

(2) Let $\mathcal{A}$ be a poset and $\mathcal{F}=\left(Q_{a}: a \in \mathcal{A}\right)$ a family of subgroups of a group $G$. Assume $\mathcal{F}$ is compatible; that is $Q_{a} \leq Q_{b}$ for all $a, b \in \mathcal{A}$ with $a \leq b$. Define $P(\mathcal{F})$ to be the poset consisting of the disjoint union of the coset spaces $G / Q_{a}$ with $Q_{a} g \leq Q_{b} h$ if and only if $a \leq b$ and $Q_{a} g \subseteq Q_{b} h$. Then visibly 
(3.1) $G$ is represented as a upper admissible group of automorphisms of $P(\mathcal{F})$ by right multiplication.

We have a partial converse to Lemma 3.1:

(3.2) Let $G$ be represented as a upper admissible group of automorphisms of $P$ transitive on 0 -cells, let $y$ be a 0 -cell of $P, \mathcal{A}=P(\geq y)$, and for $a \in \mathcal{A}$ let $Q_{a}=G_{a}$ and $\mathcal{F}=\left(Q_{a}: a \in \mathcal{A}\right)$. Then $\mathcal{A}$ is a set of representatives for the orbits of $G$ on $P$ and the map ag $\mapsto Q_{a} g$ is a $G$-equivariant isomorphism of $P$ with $P(\mathcal{F})$.

Proof. As $P$ is of finite height, each cell is above a 0 -cell, so as $G$ is transitive on 0 -cells, each cell is conjugate to a member of $\mathcal{A}$. Then as $G$ is upper admissible, $\mathcal{A}$ is a set of representatives for the orbits of $G$ on $P$.

Let $\varphi: P \rightarrow P(\mathcal{F})$ be the map $\varphi: a g \mapsto Q_{a} g$. As $\mathcal{A}$ is a set of representatives for the orbits of $G$ on $P$ and $Q_{a}=G_{a}$, the map $\varphi$ is a well defined bijection. Next $a g \leq b h$ iff $a \leq b h g^{-1}$. So if $a g \leq b h$ then $y \leq b, b h g^{-1}$, so $b=b h g^{-1}$ and hence $b h=b g$ so that $Q_{b} h=Q_{b} g$. Also $a g \leq b h=b g$ so $a \leq b$ and hence $\varphi(a g)=Q_{a} g \leq Q_{b} g=Q_{b} h=\varphi(b h)$. Similarly we can reverse the direction of the argument, and so conclude $\varphi$ is an isomomorphism of posets.

For $a \in \mathcal{A}$, let $\mathcal{F}_{a}=\left(Q_{b}: b<a\right)$ be the compatible family of subgroups of $Q_{a}$ indexed by the poset $\mathcal{A}(<a)$.

(3.3) Let $\mathcal{F}=\left(Q_{a}: a \in \mathcal{A}\right)$ be a compatible family of subgroups of a group $G$ indexed by a poset $\mathcal{A}$, let $P=P(\mathcal{F}), a \in \mathcal{A}$, and $x=Q_{a} \in P$. Then

(1) $P(>x)=\left\{Q_{b}: a<b\right\}$ is isomorphic to $\mathcal{A}(>a)$.

(2) $P(<x) \cong P\left(\mathcal{F}_{a}\right)$.

(3) $P$ is connected iff $\mathcal{A}$ is connected and $G=\langle\mathcal{F}\rangle$.

(4) $P$ is residually connected iff $\mathcal{A}$ is residually connected, $G=\langle\mathcal{F}\rangle$, and $Q_{a}=\left\langle\mathcal{F}_{a}\right\rangle$ for each $a \in \mathcal{A}$ of height at least 2 .

Proof. First $x<Q_{b} g$ iff $a \leq b$ and $Q_{a} \subseteq Q_{b} g$ iff $Q_{b} g=Q_{b}$ and $a \leq b$, so (1) holds. Simliarly $Q_{b} g<x$ iff $b<a$ and $Q_{b} g \subseteq Q_{a}$ iff $b<a$ and $g \in Q_{a}$, so (2) holds.

Let $\mathcal{A}_{i}, i \in I$, be the connected components of $\mathcal{A}, H_{i}=\left\langle Q_{a}: a \in \mathcal{A}_{i}\right\rangle$, and

$$
\Delta_{i}=\bigcup_{a \in \mathcal{A}_{i}} Q_{a} H_{i}
$$

Now for $a \in \mathcal{A}_{i}$, we just saw that $Q_{a}$ is adjacent to $Q_{b}$ for $a \leq b$ and to $Q_{c} g$ for $c<a$ and $g \in Q_{a}$, so $\Delta_{i}$ contains all cells adjacent to $Q_{a}$. Further $Q_{a}$ acts on the connected component $\Gamma(a)$ of $P$ containing $Q_{a}$ and $\left\{Q_{a}: a \in \mathcal{A}_{i}\right\} \cong \mathcal{A}_{i}$ 
is connected, so $\Gamma(a)=\Gamma(b)=\Gamma(i)$ for $a, b \in \mathcal{A}_{i}$, so $H_{i}$ acts on $\Gamma(i)$. Thus as $\Delta_{i}$ is the union of $H_{i}$ orbits of cells in $\mathcal{A}_{i}, \Delta_{i} \subseteq \Gamma(i)$ and then as all cells adjacent to $Q_{a}$ are contained in $\Delta_{i}$, we conclude $\Delta_{i}=\Gamma(i)$. Therefore $P$ is connected iff $\mathcal{A}$ is connected and $G=Q_{a} G=Q_{a} H_{i}=H_{i}$. That is (3) holds. Finally parts (1)-(3) imply part (4).

\section{Thin complexes and posets.}

In this section $K$ is an $n$-dimensional simplicial complex. We say $K$ is thin if each simplex of $K$ is contained in a chamber and for each $(n-1)$-simplex $s$ of $K, \operatorname{Link}_{K}(s)$ is of order 2. If $\operatorname{Link}(s)$ is of order 2, a reflection through $s$ is an automorphism of $K$ fixing each vertex of $s$ and interchanging the two vertices of $\operatorname{Link}(s)$.

Let $P$ be a poset of finite height $n$. The poset is thin if its order complex is thin.

Remark. (1) Thin residually connected posets of height $n$ are called abstract n-polytopes; see [MS] for example. An abstract polytope is regular if it possesses a chamber transitive group of automorphisms. Regular abstract polytopes have been studied extensively; eg. [MS]. We will study abstract polytopes with a weaker symmetry condition: a lower admissible group of automorphisms transitive on $n$-cells. For if $G$ is transitive on chambers of a poset $P$ then by 2.3 .1 and Example 1 in Section 3, $G$ is lower admissible and transitive on $n$-cells of $\operatorname{sd}(\mathcal{O}(P))$.

If $P$ is thin and $x$ is an $(n-1)$-cell, then there are $2 n$-cells above $x$, and a reflection through $x$ is an automorphism of $P$ fixing $P(\leq x)$ pointwise and interchanging the two $n$-cells above $x$. Similarly we define reflections through 1-cells dually.

(4.1) Let $K$ be thin with a connected chamber graph and $G$ a group of automorphisms of $K$. Then

(1) $G_{C}=1$ for each chamber $C$ of $K$.

(2) For each simplex $s$ of $K, G_{s}$ acts faithfully on $\operatorname{Link}_{K}(s)$.

(3) If $s$ is an $(n-1)$-simplex of $K$ then there is at most one reflection through $s$ and either $G_{s}=1$ or $G_{s}=\left\langle r_{s}\right\rangle$, where $r_{s}$ is the reflection through s.

Proof. As $K$ is thin with a connected chamber graph, a standard argument establishes (1). Then (1) implies (2). If $s$ is an $n-1$-simplex, then as $K$ is thin, $\left|\operatorname{Link}_{K}(s)\right|=2$. Then as $G_{s}$ is faithful on $\operatorname{Link}_{K}(s)$ by (2), (3) holds.

(4.2) Let $P$ be thin poset of height $n$ with a connected chamber graph, $G$ 
a group of automorphisms of $P, z$ an $n$-cell of $P, \mathcal{W}$ the set of $(n-1)$-cells below $z$, and $O(P)$ the group generated by all reflections through $(n-1)$-cells of $P$. Then

(1) If $G_{P(\leq w)} \neq 1$ for each $w \in \mathcal{W}$ then $O(P)=\left\langle r_{w}: w \in \mathcal{W}\right\rangle \leq G$ and $O(P)$ is transitive on the $n$-cells of $P$.

(2) If $G$ is lower admissible and transitive on the $n$-cells of $P$ then $G_{P(\leq w)}$ $\neq 1$ for each $w \in \mathcal{W}$ and $G=O(P)$.

Proof. Let $w \in \mathcal{W}$. As $P$ is thin there is exactly one more $n$-cell $y$ above $w$. If $G$ is transitive on $n$-cells there is $g \in G$ with $z g=y$. Therefore

$$
P(\leq w) \subseteq P(\leq z) \cap P(\leq z g),
$$

so if $G$ is lower admissible then $g \in G_{P(\leq w)}$, so $G_{P(\leq w)} \neq 1$, proving half of (2).

Conversely assume $G_{P(\leq w)} \neq 1$ for each $w \in \mathcal{W}$. Then by 4.1.3 applied to $K=\mathcal{O}(P), G_{P(\leq w)}=\left\langle r_{w}\right\rangle \cong \mathbf{Z}_{2}$, where $r_{w}$ is the unique reflection through $w$ and $z r_{w}=y$. Let $H=\left\langle r_{w}: w \in \mathcal{W}\right\rangle$. To complete the proof of (2) and to establish (1), it remains to show $H=O(P)$ is transitive on $n$-cells and if $G$ is lower admissible then $H=G$. If $H$ is transitive on $n$-cells and $G$ is lower admissible then $G=H G_{z}=H G_{P(\leq z)}=H$, since by 4.1.1, $G_{P(\leq z)}=1$. Further as $H$ contains each reflection through a member of $\mathcal{W}$, and as $H$ is transitive on $n$-cells, $H$ contains all reflections, so $H=O(P)$. Therefore it remains to show $H$ is transitive on $n$-cells.

Let $\Gamma$ be the graph on the $n$-cells of $P$ with $a$ adjacent to $b$ if there is a common $(n-1)$-cell below $a$ and $b$. As the chamber graph of $P$ is connected, so is $\Gamma$. Further if $w<y, z$ is an $(n-1)$-cell then $z r_{w}=y$. That is if $y$ is adjacent to $z$ then $y \in z H$. Now if $H$ is not transitive on $n$-cells, there exists $y$ at minimal distance $d$ from $z$ subject to $y \notin z H$. By minimality of $d$ there is $x$ adjacent to $y$ with $x \in z H$. Then as $u \in z H$ for each $u$ adjacent to $z$ and $x \in z H, y \in x H=z H$, contrary to the choice of $y$. This completes the proof of the lemma.

(4.3) Let $\mathcal{A}$ be a poset of height $n$ with a unique 0-cell $a_{0}$ and $\mathcal{F}=\left(Q_{a}\right.$ : $a \in \mathcal{A})$ a compatible family of subgroups of a group $G$. Then $P(\mathcal{F})$ is an abstract polytope if and only if the following conditions hold:

(1) $Q_{a_{0}}=1$.

(2) For each 1-cell a of $\mathcal{A}, Q_{a}$ is of order 2.

(3) $\mathcal{A}\left(>a_{0}\right)$ is an abstract polytope.

(4) $Q_{b}=\left\langle Q_{a}: a \leq b\right.$ and $\left.h(a)=1\right\rangle$ for each $b \in \mathcal{A}$.

(5) $\quad G=\langle\mathcal{F}\rangle$. 
Proof. Let $P=P(\mathcal{F})$ and $Q_{0}=Q_{a_{0}}$. By 3.3, $P$ is residually connected iff $\mathcal{A}$ is residually connected, $G=\langle\mathcal{F}\rangle$, and $Q_{b}=\left\langle\mathcal{F}_{b}\right\rangle$ for each $b \in \mathcal{A}$ of height at least 2. Notice the last condition is equivalent to (4) when $Q_{0}=1$.

Next $\mathcal{A} \cong P\left(\geq Q_{0}\right)$ by 3.3 , so if $P$ is an abstract polytope then (3) holds. By 4.1.1, $Q_{0}=1$. Further if $a$ is a 1-cell in $\mathcal{A}$ then by 3.3, $P(<$ $\left.Q_{a}\right) \cong Q_{a} / Q_{0} \cong Q_{a}$ is of order 2 , so (2) holds. Thus (1)-(5) are necessary conditions.

Conversely assume (1)-(5); then we have seen that $P$ is residually connected. Next if $Q_{a} g \leq Q_{b} h$ with $h\left(Q_{b} h\right)=h\left(Q_{a} g\right)+2$ then $a<b$ with $h(a)=h(b)+2$ and by 3.3 , the intervals $\left(Q_{a} g, Q_{b} h\right)_{P} \cong(a, b)_{\mathcal{A}}$ are of order 2 by (3). If $Q_{b} h$ is a 1-cell then $b$ is a 1 -cell and $P\left(<Q_{b} h\right) \cong Q_{b} / Q_{0}$ is of order 2 by (1) and (2). Finally if $Q_{b} g$ is an $n-1$-cell then by 3.3, $P\left(>Q_{b} g\right) \cong \mathcal{A}(>b)$ is of order 2 by (3). So $P$ is thin and (1)-(5) are sufficient.

Let $I$ be a finite index set and $\rho: I \rightarrow G$ a map such that $\rho(i)$ is an involution for each $i \in I$. For $J \subseteq I$ define

$$
P_{J}=\langle\rho(j): j \in J\rangle .
$$

The groups $P_{J}, J \subseteq I$, are the parabolics of the pair $(G, \rho)$.

Define the Coxeter matrix of $(G, \rho)$ to be the matrix $M=\left(m_{i j}\right)$ defined by $m_{i j}=|\rho(i) \rho(j)|$ and the Coxeter diagram $\Delta$ of $(G, \rho)$ to be the Coxeter diagram of its Coxeter matrix. That is $\Delta$ is the diagram whose nodes are the elements of $I$ and with an edge of weight $m_{i, j}-2$ between distinct nodes $i$ and $j$.

We have a graph induced on $I$ by the Coxeter diagram $\Delta$ via $i$ adjacent to $j$ if there is an edge between $i$ and $j$ of weight at least 1 ; i.e. if $m_{i, j}>2$. We say $\Delta$ is connected if its graph is connected, and more generally define the connected components of $\Delta$ to be the connected components of its graph.

Define a thin triple of height $n$ to be a triple $(G, \rho, \mathcal{A})$ such that

(T1) $G$ is a group.

(T2) $\mathcal{A}$ is a poset of height $n$ with a unique element $a_{0}$ of height $0, \mathcal{A}\left(>a_{0}\right)$ is thin, and $\mathcal{A}\left(>a_{0}\right)$ is residually connected if $n>1$.

(T3) Let $I$ be the set of 1 -cells of $\mathcal{A}$. Then $\rho: I \rightarrow G$ is a function with $\rho(i)$ an involution for each $i \in I$ and $G=\langle\rho(I)\rangle$.

Let $\mathcal{F}=\mathcal{F}(G, \rho, \mathcal{A})$ be the family of subgroups $\mathcal{F}=\left(Q_{a}: a \in \mathcal{A}\right)$ with $Q_{a}=P_{I(a)}$ the parabolic determined by $I(a)=\{i: i \leq a\}$. The family $\mathcal{F}$ is the family of parabolics of $(G, \rho, \mathcal{A})$ and the poset $P(\mathcal{F})$ is the parabolic poset of $(G, \rho, \mathcal{A})$ and is denoted by $P(G, \rho, \mathcal{A})$.

(4.4) Let $(G, \rho, \mathcal{A})$ be a thin triple, $\mathcal{F}=\left(Q_{a}: a \in \mathcal{A}\right)$ the parabolic family and $P=P(\mathcal{F})$ the parabolic poset of $(G, \rho, \mathcal{A})$. Then

(1) $P$ is an abstract polytope and $G$ is an upper admissible group of auto- 
morphisms of $P$ transitive on 0 -cells of $P$.

(2) $\mathcal{F}$ is a set of representatives for the orbits of $G$ on $P$ and for $a \in \mathcal{A}$, $h\left(Q_{a}\right)=h(a)$ and $Q_{a}$ is the stablizer in $G$ of the cell $a$.

(3) For each $i \in I, \rho(i)$ is the reflection through the 1-cell $Q_{i}$ of $P$.

Proof. By definition, $Q_{0}=Q_{a_{0}}=P_{I\left(a_{0}\right)}=P_{\varnothing}=1$ and for $i \in I, Q_{i}=$ $P_{i}=\langle\rho(i)\rangle$ is of order 2. By hypothesis, $\mathcal{A}\left(>a_{0}\right)$ is an abstract polytope and $G=\langle\mathcal{F}\rangle$. By construction $Q_{a}=P_{I(a)}=\left\langle Q_{i}: i \leq a\right.$ and $\left.h(i)=1\right\rangle$. Therefore by $4.3, P$ is an abstract polytope. By 3.1, $G$ is an upper admissible group of automorphisms of $P$ and by construction (2) holds so as $a_{0}$ is the unique 0 -cell in $\mathcal{A}, G$ is transitive on 0 -cells. Finally part (3) holds by the dual of 4.1.3.

(4.5) Let $P$ be an abstract polytope, $a_{0}$ a 0 -cell of $P$, and $G$ an upper admissible group of automorphisms of $P$ transitive on 0 -cells. Let $\mathcal{A}=P(\geq$ $\left.a_{0}\right), I$ the set of 1-cells in $\mathcal{A}$, and $\rho(i)$ the reflection through $i \in I$. Then

(1) $G=O(P)$ is the group generated by all reflections through 1-cells of $P$.

(2) $(G, \rho, \mathcal{A})$ is a thin triple and $P \cong P(G, \rho, \mathcal{A})$ via the $G$-equivariant map $a g \mapsto Q_{a} g$.

(3) $Q_{a}=O(P(<a))$ for each $a \in \mathcal{A}$.

Proof. Part (2) follows from 3.2 and part (1) from the dual of 4.2.2. For $a \in \mathcal{A}, P(<a)$ is an abstract polytope and $Q_{a}=G_{a}$ is transitive on 0-cells of $P(<a)$, so (1) implies (3).

Remark. (1) Theorems 4.4 and 4.5 show the notion of a thin triple $(G, \rho, \mathcal{A})$ and the notion of an abstract polytope admitting $G$ as an upper admissible group of automorphisms transitive on 0-cells are equivalent. Namely the map $(G, \rho, \mathcal{A}) \mapsto P(G, \rho, \mathcal{A})$ induces a bijection between the collection of isomorphism classes of thin triples and the collection of isomorphism classes of abstract polytopes with an upper admissible group of automorphisms transitive on 0-cells. The inverse of this bijection is induced by $P \mapsto(O(P), \rho, \mathcal{A})$, where $a_{0}$ is some fixed 0-cell of $P, \mathcal{A}=P\left(\geq a_{0}\right)$ and $\rho(i)$ is the reflection through $i$.

\section{Generalities on triangulations.}

Let $\mathbf{R}^{n}$ be $n$-dimensional Euclidean space. An affine subspace of $\mathbf{R}^{n}$ is a coset $U+x$ of a linear subspace $U$ of $\mathbf{R}^{n}$. The dimension of the affine subspace $U+x$ is $\operatorname{dim}(U)$, with the empty set of dimension -1 . 
A subset $C$ of $\mathbf{R}^{n}$ is convex if for each $x, y \in C$ and each real number $t$ with $0 \leq t \leq 1, t x+(1-t) y \in C$. The intersection of any family of convex sets is convex, so for each subset $S$ if $\mathbf{R}^{n}$ there is a smallest convex subset $[S]$ of $\mathbf{R}^{n}$ containing $S$. We call $[S]$ the convex closure of $S$. Recall that if $S=\left\{v_{0}, \ldots, v_{k}\right\}$ then

$$
[S]=\left\{\sum_{i=0}^{k} a_{i} v_{i}: 0 \leq a_{i} \in \mathbf{R}, \text { and } \sum_{i} a_{i}=1\right\}
$$

with this expression being unique if $S$ is affine independent; that is if the affine subspace generated by $S$ is of affine dimension $k$.

Let $K$ be a finite dimensional simplicial complex with vertex set $K$ and simplices $\Sigma$. A triangulation of a topological space $T$ by $K$ is a map $\varphi$ of $\Sigma$ into the set of closed subspaces of $T$ together with homeomorphisms

$$
\varphi_{s}: \varphi(s) \rightarrow \hat{\varphi}(s)=[u(s, v): v \in s] \subset \mathbf{R}^{k}
$$

for each $k$-simplex $s$ of $K$ such that:

(T1) For $s, t \in \Sigma, \varphi(s) \cap \varphi(t)=\varphi(s \cap t)$, where $\varphi(\varnothing)=\varnothing$.

(T2) $T=\bigcup_{s \in \Sigma} \varphi(s)$ and $C \subseteq T$ is closed in $T$ if and only if $C \cap \varphi(s)$ is closed in $\varphi(s)$ for all $s \in \Sigma$.

(T3) For each $k$-simplex $s$ of $K$ and $t \subseteq s, \hat{\varphi}(s)=[u(s, v): v \in s]$ is of affine dimension $k$ and $\varphi_{t, s}=\varphi_{s} \circ \varphi_{t}^{-1}$ acts on $\hat{\varphi}(t)=[u(t, v): v \in t]$ via $\varphi_{t, s}: \sum_{v \in t} a_{v} u(t, v) \mapsto \sum_{v \in t} a_{v} u(s, v)$.

A morphism of topological spaces $\varphi^{i}: K^{i} \rightarrow T^{i}, i=1,2$, with triangulation is a pair $(\alpha, \beta)$ where $\alpha: K^{1} \rightarrow K^{2}$ is a simplicial map, $\beta: T^{1} \rightarrow T^{2}$ is continuous, and for each $s \in \Sigma^{1}$,

$\left(T_{1}\right) \beta\left(\varphi^{1}(s)\right) \subseteq \varphi^{2}(\alpha(s))$, and

$\left(T_{2}\right) \alpha_{s} \circ \varphi_{s}^{1}=\varphi_{\alpha(s)}^{2} \circ \beta$, where $\alpha_{s}: \hat{\varphi}^{1}(s) \rightarrow \hat{\varphi}^{2}(\alpha(s))$ is defined by

$$
\alpha_{s}: \sum_{v \in s} a_{v} u(s, v) \mapsto \sum_{v \in s} a_{v} u(\alpha(s), \alpha(v)) .
$$

Any pair $\varphi_{i}: K \rightarrow T_{i}, i=1,2$, of triangulations of topological spaces $T_{i}$ by $K$ are isomorphic and there exists a canonical triangulation $\varphi: K \rightarrow T=$ $T(K)$ called the geometric realization of $K$. Moreover $T$ extends to a functor from the category of simplicial complexes to the category of triangulated topological spaces. Namely if $\alpha: K \rightarrow \bar{K}$ is a simplicial map, then $\alpha$ extends to a map $T(\alpha): \varphi \rightarrow \bar{\varphi}$. In particular this shows that $\operatorname{Aut}(K) \cong \operatorname{Aut}(\varphi)$.

Let $\beta$ be a homeomorphism of $T=T(K)$ permuting the cells $\varphi(s), s \in \Sigma$. Then $\beta$ induces a permutation $\alpha: \Sigma \rightarrow \Sigma$ of $\Sigma$ via $\beta(\varphi(s))=\varphi(\alpha(s))$. As $t \subseteq s$ if and only if $\varphi(t) \subseteq \varphi(s)$, the map $\alpha$ is an automorphism $\alpha: K \rightarrow K$. 
We say that $\beta$ preserves the cell structure of $T$ if $\alpha_{s} \circ \varphi_{s}=\varphi_{\alpha(s)} \circ \beta$, where $\alpha_{s}$ is the map defined in Axiom $\left(T_{2}\right)$ for morphisms of triangulations. In otherwords the set $G$ of all homeomorphims $\beta$ preserving the cell structure on $T$ forms a group and the maps

$$
\alpha \mapsto(\alpha, \beta) \mapsto \beta
$$

are isomorphisms $\operatorname{Aut}(K) \cong \operatorname{Aut}(\varphi) \cong G$. Thus we can regard the automorphism group of the triangulated space $T$ to be the group of all homeomorphisms preserving the cell structure on $T$, and that group is naturally isomorphic to $\operatorname{Aut}(K)$.

Finally define an automorphism $\beta$ of the triangulated space $T$ to be $a d$ missible if whenever $\beta$ acts on a cell $\varphi(s)$ then $\beta$ induces the identity map on $\varphi(s)$ and define $\beta$ to be strongly admissible if $\beta$ induces the identity on $\varphi(s) \cap \varphi(s) \beta$ for each cell $\varphi(s)$. Notice that $\beta$ is admissible if and only if the automorphism $\alpha$ of $K$ corresponding to $\beta$ is an admissible automorphism of $K$ and $\beta$ is strongly admissible if and only if $\alpha$ is strongly admissible.

\section{Manifolds.}

Let $n$ be a nonnegative integer. A topological n-manifold is a nonempty Hausdorff space $T$ which is locally isomorphic to $n$-dimensional Euclidean space; that is each point of $T$ has a neighborhood homeomorphic to a neighborhood of $\mathbf{R}^{n}$. Let $R=\mathbf{Z}$ or a field. A $R$-homology $n$-manifold is a nonempty Hausdorff space $T$ such that for each point $t \in T$,

$$
H_{i}(T, T-\{t\}, R) \cong\left\{\begin{array}{ll}
0 & \text { if } i \neq n \\
R & \text { if } i=n
\end{array}\right\} .
$$

We say $T$ is a homology $n$-manifold if $R=\mathbf{Z}$ and $T$ is a $p$-homology $n$ manifold if $R$ is a field of prime characteristic $p$.

(6.1) Each topological n-manifold $T$ is a homology n-manifold.

Proof. See Lemma 35.1 and Example 1 in Section 35 of [M].

Let $K$ be a simplicial complex. We say $K$ is a $R$-homology $n$-sphere if $\operatorname{dim}(K)=n$ and $K$ has the $R$-homology of an $n$-sphere. That is $\tilde{H}_{i}(K, R)=$ 0 for $i \neq n$ and $\tilde{H}_{n}(K, R) \cong R$. A $R$-homology (-1)-sphere is an empty simplicial complex. We define $K$ to be a $R$-homology $n$-manifold if $\operatorname{Link}_{K}(s)$ is a $R$ - homology $(n-k-1)$-sphere for each $k$-simplex $s$ and each $0 \leq k<n$. For $s$ a simplex of $K$ and $\varphi: K \rightarrow T(K)$ the geometric realization of $K$, let

$$
I(s)=\varphi(s)-\bigcup_{t \subset s} \varphi(t)
$$


be the interior of $\varphi(s)$.

(6.2) Let $K$ be a finite dimensional simplicial complex. Then

(1) $K$ is a $R$-homology $n$-manifold if and only if the geometric realization $T$ of $K$ is a $R$-homology n-manifold.

(2) Assume $K$ is a connected $R$-homology $n$-manifold. Then $K$ is thin and residually connected. Further $\operatorname{Link}_{K}(s)$ is finite for each simplex $s$ of $K$.

Proof. If the geometric realization $T$ of $K$ is a $R$-homology $n$-manifold then by Theorem 63.2 in $[\mathbf{M}], K$ is a $R$-homology $n$-manifold. Conversely assume $K$ is a $R$-homology $n$-manifold and let $x \in T$. Then $x \in I(s)$ for a unique simplex $s$ of $K$. Let $k=\operatorname{dim}(s)$. If $k=n$ then

$$
I(s)=T-\bigcup_{s \neq t \in \Sigma(K)} \varphi(t)
$$

so $I(s)$ is open in $T$ and as $\varphi(s)$ is homeomorphic to a closed $n$-ball, $I(s) \cong$ $\mathbf{R}^{n}$. So $I(s)$ is a neighborhood of $T$ homeomorphic to $\mathbf{R}^{n}$, and hence by Theorem 35.1 and Example 1 in Section 35.1 of $[\mathbf{M}], H_{i}(T, T-\{x\}, R)$ is isomorphic to $R$ or 0 for $i=n, i \neq n$, respectively.

So assume $k<n$. Then by Lemma 63.1 in [M],

$$
H_{i}(T, T-\{x\}, R) \cong \tilde{H}_{i-k-1}\left(\operatorname{Link}_{K}(s), R\right)
$$

so that $H_{i}(T, T-\{x\}, R)$ is 0 unless $i=n$, where it is $R$.

So (1) is established. Thus we may assume $K$ is a connected $R$-homology $n$-manifold. Then $H_{0}\left(\operatorname{Link}_{K}(s), R\right) \cong R$ so $\operatorname{Link}_{K}(s)$ is connected for each $k$ - simplex $s$ such that $0 \leq k<n-1$, while $\operatorname{Link}_{K}(s)$ is of order 2 if $k=n-1$. Therefore $K$ is residually connected and thin of dimension $n$. Finally by Exercise 4 on page 377 of $[\mathrm{M}], \operatorname{Link}_{K}(s)$ is finite for each simplex $s$, completing the proof of (2).

Suppose $K$ is finite of dimension $n$. We say $K$ is $R$-orientable if there exists an orientation of the $n$ - simplices of $K$ such that

$$
\sum_{s \in \Sigma^{n}(K)} s
$$

is a cycle in $C_{n}(K, R)$.

(6.3) If $K$ is a connected $R$-homology $n$ - manifold with $H_{n}(K, R) \neq 0$ then $K$ is finite and orientable and $H_{n}(K, R) \cong R$.

Proof. If $H_{n}(K, R) \neq 0$ then by Exercise 4 on page 377 of $[\mathbf{M}], K$ is finite and then Corollary 65.3 in $[\mathbf{M}]$ completes the proof. 
(6.4) Let $K$ be a finite dimensional simplicial complex which is a p-homology $n$-sphere for some prime $p$ and $G$ a p-group acting strongly admissibly on $K$. Then

(1) $\operatorname{Fix}_{K}(G)$ is a p-homology $r$-sphere for some $-1 \leq r \leq n$.

(2) If $K$ is a homology p-manifold then $r=\operatorname{dim}\left(\operatorname{Fix}_{K}(G)\right)$ and either $r=-1$ and $\operatorname{Fix}_{K}(G)=\varnothing$, or $r=0$ and $\operatorname{Fix}_{K}(G)$ is disconnected of order 2, or $r>0$ and $\operatorname{Fix}_{K}(G)$ is a connected p-homology $r$ - manifold.

Proof. Part (1) is a special case of Smith's Theorem. If $G$ is topologically regular on $K$ it appears as Theorem 5.1 in $[\mathrm{Br}]$, so it remains to reduce to the case $G$ topologically regular. But by $2.2, K$ and $s d^{2}(K)$ have the same homotopy type, $G$ is topologically regular on $s d^{2}(K)$, and $\operatorname{Fix}_{s d^{2}(K)}(G)=$ $s d^{2}\left(\operatorname{Fix}_{K}(G)\right)$. By the first remark, $s d^{2}(K)$ is a $p$-homology $n$-sphere, so by the second remark and Smith's Theorem, $\operatorname{Fix}_{s d^{2}(K)}(G)$ is a $p$-homology $r$ sphere, and then by the first and third remarks, $\operatorname{Fix}_{K}(G) \simeq s d^{2}\left(\operatorname{Fix}_{K}(G)\right)=$ $\operatorname{Fix}_{s d^{2}(K)}(G)$ is too.

Similarly (2) is well known and can be retrieved using Theorem 2.2 in Chapter 5 of $[\mathbf{B o}]$. Alternatively $[\mathbf{A}]$ contains a sketch of a proof of this fact in our special situation, following Bredon in Theorem 2.1 in Chapter IV of $[\mathrm{Br}]$.

(6.5) Let $K$ be a homology n-manifold. Then:

(1) $K$ is a p-homology manifold for each prime $p$.

(2) If $K$ is a homology $n$-sphere then $K$ is a p-homology $n$-sphere for each prime $p$.

Proof. Notice (2) implies (1), so assume $K$ is a homology sphere. Let $\tilde{C}_{*}$ be the augmented chain complex for $K$. Thus $\tilde{H}_{k}(K)$ is 0 if $k \neq n$ and $\mathbf{Z}$ if $k=n$. Let $x \in \tilde{C}_{k}$ with $\partial(x)=p y$ for some $y \in \tilde{C}_{k-1}$. Then $0=\partial^{2}(x)=$ $\partial(p y)=p \partial(y)$, so $y \in \tilde{Z}_{k-1}(K)$ and hence there is $z \in \tilde{C}_{k}$ with $\partial(z)=y$. Then $0=\partial(x-p z)$, so if $k \neq n$ there is $u \in \tilde{C}_{k+1}$ with $\partial(u)=x-p z \equiv x$ $\bmod p \tilde{C}_{k}$. Hence $\tilde{H}_{k}\left(K, \mathbf{F}_{p}\right)=0$ for $k \neq n$, so it remains to show the top homology is 1-dimensional.

Now relax the assumption that $K$ is a homology manifold and sphere, and assume instead that $K$ is thin and residually connected. Let

$$
x=\sum_{s \in \Sigma^{n}(K)} a_{s} s \in \tilde{C}_{n} .
$$

Then

$$
\partial(x)=\sum_{s, i}(-1)^{i} a_{s} s^{i}=\sum_{t \in \Sigma^{n-1}(K)}\left(\epsilon_{t, s} a_{s}+\epsilon_{t, s^{\prime}} a_{s^{\prime}}\right) t
$$


where $s, s^{\prime}$ are the $n$-simplices above the $(n-1)$-simplex $t$ and $\epsilon_{t, s}=(-1)^{i}$, where $t=s^{i}$. Thus $\partial(x) \in p \tilde{C}_{n-1}$ if and only if

$$
a_{s^{\prime}} \equiv-\epsilon_{t, s} \epsilon_{t, s^{\prime}} a_{s} \quad \bmod p
$$

for all $t \in \Sigma^{n-1}(K)$. Hence as the graph on $\Sigma^{n}(K) \cup \Sigma^{n-1}(K)$ with $s$ adjacent to $t$ if $t \subset s$, is connected, we conclude that if $K$ is a homology $n$ - sphere then $\partial(x) \in p \tilde{C}_{n-1}$ if and only if $x \in p \mathbf{Z}+\pi$, where $\pi=\sum_{s} c_{s} s$ is the fundamental cycle with $c_{s}= \pm 1$ for all $s$ and $\epsilon_{t, s} c_{s}+\epsilon_{t, s^{\prime}} c_{s^{\prime}}=0$ for all $t \in \Sigma^{n-1}(K)$. This completes the proof of (2). Further if $K$ is only thin and residually connected, we see that $\pi=\sum_{s} s$ is a cycle over $\mathbf{F}_{2}$, so we have shown:

If $K$ is thin and residually connected then $K$ is $\mathbf{F}_{2}$-orientable.

\section{Combinatorial cell complexes.}

The notion of a combinatorial cell complex $(X, f)$ is defined in $[\mathbf{C C C}]$. We will consider only the case where $(X, f)$ is the simplicial cell complex of the poset $X$. That is $X$ is a poset in which each element is of finite height and for $x \in X, f(x)=X(\leq x)$. The posets $f(x), x \in X$, are the cells of $(X, f)$ and the boundary of the cell $f(x)$ is $\dot{f}(x)=f(x)-\{x\}$. The faces of the cell are its subposets $f(x)(\leq v), v \in f(x)$.

From Section 5, there is a functor $T$ from the category of posets to the category of topological spaces which associates to $X$ its geometric realization $T(X)=T(\mathcal{O}(X))$. Further $T(X)$ is the union of the closed subspaces $T(x)=$ $T(f(x)), x \in X$, which supply $T(X)$ with a cell structure parametrized by $X$. A topological space with cell structure over $X$ is just a space homeomorphic to $T(X)$. By construction

(7.1) There is a triangulation $\varphi: \mathcal{O}(X) \rightarrow T(X)$ of the geometric realization of $X$ by $\mathcal{O}(X)$.

Because of 7.1, the homology, cohomolgy, and fundamental group of the geometric realization $T(X)$ of $X$ are the same as that of $\mathcal{O}(X)$ (defined combinatorially), and we define $H_{*}(X)=H_{*}(\mathcal{O}(X)), H^{*}(X)=H^{*}(\mathcal{O}(X))$, and $\pi_{1}(X)=\pi_{1}(\mathcal{O}(X))$.

Recall a space with cell structure over $X$ is just a copy of $T(X)$, so we can regard any such space as $T(X)$. An automorphism of a space with cell structure is an automorphism $\alpha$ of the triangulated space $\varphi: \mathcal{O}(X) \rightarrow T(X)$ (as defined in Section 5) permuting the cells $T(x), x \in X$. In particular from Section 5, $\alpha$ is induced from a unique automorphism of $\mathcal{O}(X)$ and then as $\alpha$ permutes the cells $T(x), x \in X, \alpha$ preserves height, so $\alpha$ is induced by an automorphism of $X$. That is the group of automorphisms of a space with cell structure over $X$ is isomorphic to $\operatorname{Aut}(X)$. 
We next discuss cellular homology. Define the $n$-skeleton of $X$ to be

$$
X^{n}=\{x \in X: h(x) \leq n\} .
$$

Let $\mathcal{O}^{n}=\mathcal{O}\left(X^{n}\right)$ be the order complex of the $n$-skeleton of $X$. If $s$ is a $k$-simplex of $K=\mathcal{O}(X)$ then order $s=\left\{v_{0}, \ldots, v_{k}\right\}$ so that $v_{i} \leq v_{i+1}$ for each $i$. Use this ordering of $s$ to define the oriented simplex

$$
s=v_{0} \cdots v_{k}=v_{0} \wedge \cdots \wedge v_{k}
$$

Then these oriented simplices are generators for $C_{k}(K)$ the $k$ th member of the simplical chain group $C_{*}(K)=\left(C_{n}(K): n\right)$ with boundary map $\partial$ defined by

$$
\partial(s)=\sum_{i=0}^{k}(-1)^{i} s^{i}
$$

where $s^{i}=v_{0} \wedge \cdots \wedge v_{i-1} \wedge v_{i+1} \wedge \cdots \wedge v_{k}$. If $h(x)=n$, a typical member of $C_{n}(f(x))$ is of the form $\sum_{s} a_{s} s x$, where the sum is over all $(n-1)$-simplices $s$ of $\dot{f}(x)$ and $a_{s} \in \mathbf{Z}$. Notice $\tilde{H}_{n-1}(\dot{f}(x))=\tilde{Z}_{n-1}(\dot{f}(x))$. Let

$$
D(x)=\left\{\sum_{s} a_{s} s x: \sum_{s} a_{s} s \in \tilde{Z}_{n-1}(\dot{f}(x))\right\} \leq C_{n}(f(x))
$$

so that $D(x) \cong \tilde{H}_{n-1}(\dot{f}(x))$, and define

$$
D_{n}(X)=\bigoplus_{h(x)=n} D(x) \cong \bigoplus_{h(x)=n} \tilde{H}_{n-1}(\dot{f}(x)) .
$$

Finally define the boundary map on the chain complex $D_{*}(X)=\left(D_{n}(X)\right.$ : $0 \leq n \in \mathbf{Z})$ by

$$
\begin{aligned}
\partial_{n}: D_{n}(X) & \rightarrow D_{n-1}(X) \\
\sum_{x, s} a_{x, s} s x & \mapsto \sum_{x, s} a_{x, s} s .
\end{aligned}
$$

We call $D_{*}(X)$ the cellular chain complex of $X$ and $\partial$ the cellular boundary map. The cellular homology of $X$ is $H_{*}^{c}(X)=H_{*}\left(D_{*}(X)\right)$.

Theorem 7.2. If $\mathcal{O}(\dot{f}(x))$ is homology spherical for each $x \in X$ then the ordinary homology $H_{*}(X)$ of $X$ is isomorphic to the cellular homology $H_{*}^{c}(X)$.

Proof. This is Theorem 12.16 in [CCC].

Define $\hat{X}$ to be the dual poset of $X$. That is $X$ and $\hat{X}$ are the same as sets with the ordering $\lesssim$ on $\hat{X}$ defined by $x \lesssim y$ if and only if $y \leq x$. Then 
for $x \in \hat{X}$, define $\hat{f}(x)=X(\geq x)$ and $\check{f}(x)=\hat{f}(x)-\{x\}$, giving rise to the dual complex $(\hat{X}, \hat{f})$ of $(X, f)$. Then of course

(7.3) $\mathcal{O}(X)=\mathcal{O}(\hat{X})$, so $X$ and $\hat{X}$ have the same homology and fundamental group.

Theorem 7.4. Let $X$ be a poset of height $n$ and $R$ be $\mathbf{Z}$ or a field. Then the following are equivalent.

(1) The geometric realization of $X$ is a $R$-homology $n$-manifold.

(2) For each $x \in X$ of height $h, \check{f}(x)$ is a R-homology $(n-h-1)$-sphere and $\dot{f}(x)$ is a R-homology $(h-1)$-sphere.

(3) For each $x \in X$ of height 0 and each $y \in X$ of height $n, \check{f}(x)$ and $\dot{f}(y)$ are both $R$-homology $(n-1)$-spheres and $R$-homology $(n-1)$-manifolds.

Proof. First we recall that the geometric realizations of $X$ and $K=\mathcal{O}(X)$ are the same, and by 6.2.1, that space is a R-homology $n$-sphere if and only if $\operatorname{Link}_{K}(s)$ is a homology $(n-k-1)$-sphere for each $k$-simplex $s$ of $K$. So let $s=\left\{v_{0}, \ldots, v_{k}\right\}$ be a $k$-simplex of $K$ with $v_{i} \leq v_{j}$ for $i \leq j$. Notice

(a) If $v_{k}$ is of height $k$ then $\operatorname{Link}_{K}(s) \cong \check{f}\left(v_{k}\right)$.

The dual of (a) is:

(b) If $v_{0}$ is of height $n-k$ then $\operatorname{Link}_{K}(s) \cong \dot{f}\left(v_{0}\right)$.

Observe that (a) and (b) together with paragraph one of this proof show that (1) implies (2). Similarly if $K$ is a R-homology manifold, so are all its links, so (a) and (b) show that (1) implies (3). Conversely

$$
\operatorname{Link}_{K}(s)=\dot{f}\left(v_{0}\right) * S_{1} * \cdots * S_{k} * \check{f}\left(v_{k}\right)
$$

is the join of $\dot{f}\left(x_{0}\right), \check{f}\left(v_{k}\right)$, and the intervals

$$
S_{i}=\check{f}\left(v_{i-1}\right) \cap \dot{f}\left(v_{i}\right)
$$

so as the join of a R-homology $p$-sphere with a R-homology $q$-sphere is a R-homology $p+q+1$-sphere, to show (2) implies (1), it suffices to show that (2) implies that $S_{i}$ is a homology $\operatorname{dim}\left(S_{i}\right)$-sphere, since $\operatorname{dim}\left(\operatorname{Link}_{K}(s)\right)=$ $n-k-1$. This follows from (2) using the argument in the next paragraph.

Finally assume (3). For $x \in X$ of height $h, x \leq y$ for some $y$ of height $n$. If $x=y$ then by hypothesis $\dot{f}(x)$ is a $R$-homology $(n-1)$-sphere. If not then as $\dot{f}(y)$ is a $R$-homology manifold, $\dot{f}(y)(<x)=\dot{f}(x)$ is a $R$-homology $(h-1)$-sphere. By duality, $\check{f}(x)$ is a $R$-homology $(n-h-1)$-sphere, so (3) implies (2).

Finally we find in Theorem 15.15 of $[\mathbf{C C C}]$ that: 
(7.5) There is an equivalence of categories between the category of regular $C W$-complexes and posets $X$ in which $\mathcal{O}(\dot{f}(x))$ is an $(n-1)$-sphere for each $x \in X$ of height $n$ and each $1 \leq n \leq h(X)$.

\section{Poincaré duality.}

In this section $R=\mathbf{Z}$ or $R$ is a field and $X$ is a poset of height $n$ such that the geometric realization $T(X)$ of $X$ is a $R$-homology $n$ - manifold. Let $K=\mathcal{O}(X)$ be the order complex and $\hat{X}$ the dual poset. We say $X$ is a $R$-homology $n$-manifold if its geometric realization is an $R$-homology $n$-manifold.

From Section 7, we have the celluar chain complex $D_{*}=D_{*}(X)$ and the homology of $X$ is isomorphic to the homology of $D_{*}$. Similarly let $\hat{D}_{*}=$ $D_{*}(\hat{X})$ be the cellular chain complex of $\hat{X}$. By $7.3, \hat{D}_{*}$ has the same homology as $D_{*}$.

Recall from Section 7 that

$$
D_{k}=\bigoplus_{h(x)=k} D(x)
$$

where $D(x) \cong \tilde{H}_{k-1}(\dot{f}(x))$. As $X$ is a $R$-homology $n$-manifold, $\tilde{H}_{k-1}(\dot{f}(x), R)$ $\cong R$ by 7.4. Thus $D(x)=R \sigma_{x}$, where $\sigma_{x}=d_{x} x$ and $d_{x}$ is a generator for $\tilde{H}_{k-1}(\dot{f}(x), R)$. Similarly $\hat{D}(x)=R \hat{\sigma}_{x}$ with $\sigma_{x}=\hat{d}_{x} x$.

Let $D^{*}$ be the cochain complex complex for $D_{*}$. Thus

$$
D^{p}(X, R)=\operatorname{Hom}\left(D_{p}(X), R\right)
$$

with the coboundary operator $\delta: D^{p}(X, R) \rightarrow D^{p+1}(X, R)$ the dual of

$$
\partial_{p}: D_{p+1}(X, R) \rightarrow D_{p}(X, R) .
$$

Following [M], write $\langle a, b\rangle$ for the image of $b \in D_{p}$ under $a \in D^{p}=$ $\operatorname{Hom}\left(D_{p}, R\right)$. We have the natural basis $\left(\sigma_{x}: h(x)=p\right)$ for $D_{p}$ and as $X$ is finite this basis is finite. Let $\left(\sigma_{x}^{*}: h(x)=p\right)$ be the dual basis for $D^{p}$. Recall the coboundary operator $\delta$ is defined by

$$
\langle\delta(a), b\rangle=\langle a, \partial(b)\rangle \quad \text { for each } a \in D^{p} \text { and } b \in D_{p+1} .
$$

The standard proof of Poincaré duality such as in Section 65 of [M] establishes the following result, which gives an explicit isomorphism between the cochain complex and the dual chain complex. Details can be found in $[\mathbf{A}]$.

Theorem 8.1. Assume $X$ is an orientable R-homology n-manifold. Then we can choose orientations for $\hat{\sigma}_{x}, x \in X$, so that the map $\phi: \sigma_{x}^{*} \mapsto \hat{\sigma}_{x}$ 
defines an isomorphism $\phi: D^{n-p} \rightarrow \hat{D}_{p}, 0 \leq p \leq n$, of the cellular cochain complex for $X$ with the dual chain complex for $X$ with $\phi \circ \delta=\partial \circ \phi$.

Corollary 8.2 (Poincaré Duality). If $X$ is an orientable R-homology $n$-manifold then $H^{p}(X, R) \cong H_{n-p}(X, R)$ for each $0 \leq p \leq n$.

Proof. By $8.1, H^{p}(X, R) \cong H_{n-p}(\hat{X}, R)$, while as remarked earlier, 7.3 says $H_{n-p}(\hat{X}, R) \cong H_{n-p}(X, R)$.

(8.3) Let $X$ be an orientable $R$-homology $n$-manifold. Then

(1) If $R$ is a field then $H_{p}(X, R) \cong H_{n-p}(X, R)$.

(2) If $R=\mathbf{Z}$ then

$$
\operatorname{Tor}\left(H_{p-1}(X)\right) \cong \operatorname{Tor}\left(H_{n-p}(X)\right) \text { and } r\left(H_{p}(X)\right)=r\left(H_{n-p}(X)\right),
$$

where $r(G)$ is the rank of $G / \operatorname{Tor}(G)$ as a $\mathbf{Z}$-module for a finitely generated abelian group $G$.

Proof. This follows from 8.2 and the Universal Coefficient Theorem; cf. 53.1 and 53.5 in $[\mathrm{M}]$.

\section{Posets as manifolds.}

In this section $R=\mathbf{Z}$ or $R$ is a field. Further $X$ is a poset of finite height $n$.

A subset $Y$ of $X$ is lower closed if $X(\leq y) \subseteq Y$ for each $y \in Y$. Define upper closed subsets dually.

(9.1) Assume $X$ is an R-homology $n$-manifold, $0<m<n, Y$ is an upper closed subset of $X$ whose minimal elements are of height $m$ in $X$, and $Z$ is a lower closed subset of $X$ whose maximal elements are of height $m$ in $X$. Assume $Y$ and $Z$ are abstract polytopes and $R$-orientable, and $Y \cap Z=\{v\}$ is of order 1 . Then $H_{m}(X, R) \neq 0$, so $X$ is not a $R$-homology sphere.

Proof. For $x \in X$, let $\sigma_{x}, \hat{\sigma}_{x}$ be the fundamental cycles for $D(x)$ and $\hat{D}(x)$ supplied in Section 8. As $Z$ is an abstract polytope, $R$-orientable, and lower closed, $\gamma=\sum_{x} c_{x} \sigma_{x} \in C_{m}(Z, R)$ is a cycle, where the sum is over those $x \in Z$ of height $m$ and $c_{x}= \pm 1$. As $Z$ is lower closed, $C_{m}(Z, R) \subseteq D_{m}(X, R)$ and the boundary map on $C_{m}(Z, R)$ is the restriction of the boundary map on $D_{m}(X, R)$, so $\gamma$ is a cycle in $D_{m}(X, R)$. We show $\gamma$ is not a boundary in $D_{m}(X, R)$ to complete the proof. Assume otherwise; then there is $\alpha \in$ $D_{m+1}(X, R)$ with $\partial(\alpha)=\gamma$.

Similarly $\hat{Y}$ is thin, residually connected, $R$-orientable, and lower closed in $\hat{X}$, so $\hat{Y}$ has a fundamental cycle $\beta$ and $\beta$ is a cycle in $D_{n-m}(\hat{X}, R)$. Now Theorem 8.1 supplies us with an isomorphism

$$
\phi: D^{m}(X, R) \rightarrow D_{n-m}(\hat{X}, R)
$$


with $\phi \circ \delta=\partial \circ \phi$ and $\phi\left(\sigma_{x}^{*}\right)=\hat{\sigma}_{x}$ for $x \in X$ of height $m$. Let $\theta=\phi^{-1}(\beta)$. Then

$$
\phi(\delta(\theta))=\partial(\phi(\theta))=\partial(\beta)=0
$$

so as $\phi$ is injective, $\delta(\theta)=0$. Hence

$$
0=\langle 0, \alpha\rangle=\langle\delta(\theta), \alpha\rangle=\langle\theta, \partial(\alpha)\rangle=\langle\theta, \gamma\rangle .
$$

Now $\beta=\sum_{x} b_{x} \hat{\sigma}_{x}$, where the sum is over those $x \in Y$ of height $m$ and $b_{x}= \pm 1$, so

$$
\theta=\phi^{-1}(\beta)=\sum_{x} b_{x} \sigma_{x}^{*}
$$

Similarly $\gamma=\sum_{x} c_{x} \sigma_{x}$ with $c_{x}= \pm 1$ and thus

$$
0=\langle\theta, \gamma\rangle=\sum_{x} b_{x} c_{x}
$$

However $Y \cap Z=\{v\}$ is of order 1 and $h(y) \geq m$ and $h(z) \leq m$ for $y \in Y$ and $z \in Z$, so $h(v)=m$. Thus $0=b_{v} c_{v}= \pm 1$, a contradiction. This completes the proof of the lemma.

Recall the notion of a thin triple from Section 4. An R-homology manifold triple of height $n$ is a thin triple $(G, \rho, \mathcal{A})$ of height $n$ such that:

(RM1) $\mathcal{A}\left(>a_{0}\right)$ is a $R$-homology manifold and $R$-homology sphere, and;

(RM2) For each $a \in \mathcal{A}$ of height at least 2, $P\left(Q_{a}, \rho_{a}, \mathcal{A}(<a)\right)$ is an $R$ homology sphere, where $\rho_{a}$ is the restriction of $\rho$ to $I(a)$.

(9.2) If $(G, \rho, \mathcal{A})$ is a $R$-homology manifold triple of height $n$ then its parabolic poset $P$ is an $R$-homology $n$-manifold and $G$ is an upper admissible group of automorphisms of $P$ transitive on 0 -cells of $P$.

Proof. By 4.4 the last two remarks hold and by 3.3.1, $\mathcal{A} \cong P\left(\geq a_{0}\right)$. Then by 7.4 , it remains to show $P(<x)$ and $P(>x)$ are $R$-homology $(n-m-1)$ and $m$-spheres, respectively, for each $x \in P$ of height $m$. But by 4.4 we may take $x=Q_{a}$ for some $a \in \mathcal{A}$, so $P(>x) \cong \mathcal{A}(>a)$ is a $R$-homology sphere by (RM1) and 3.3.1. Similarly $P(<a) \cong P\left(Q_{a}, \rho_{a}, \mathcal{A}(<a)\right)$ is a $R$-homology sphere by (RM2) and 3.3.2.

(9.3) Assume $X$ is a connected $R$-homology n-manifold and $G$ is an upper admissible group of automorphisms of $X$ transitive on 0 -cells. Let $a_{0}$ be a 0 -cell, $\mathcal{A}=X\left(\geq a_{0}\right)$, I the 1-cells in $\mathcal{A}$, and $\rho(i)$ the reflection through $i \in I$. Then $(G, \rho, \mathcal{A})$ is a $R$-homology manifold triple and $X \cong P(G, \rho, \mathcal{A})$.

Proof. By $6.2 .2, X$ is an abstract polytope. By $4.5,(G, \rho, \mathcal{A})$ is a thin triple and $X \cong P(G, \rho, \mathcal{A})$. By $7.4, \mathcal{A}\left(>a_{0}\right)$ is a $R$-homology manifold and 
sphere and $X(<a)$ is a $R$-homology sphere for each $a \in \mathcal{A}$. Then by 3.3.2, $P\left(Q_{a}, \rho_{a}, \mathcal{A}(<a)\right) \cong X(<a)$, completing the proof.

Remark. (1) Lemmas 9.2 and 9.3 show that the notion of an $R$ - homology manifold triple and the notion of a poset with an upper admissible group transitive on 0-cells, which is an $R$-homology manifold, are equivalent. Moreover 9.2 implies Theorem 2 and 4.2 and 9.3 imply Theorem 1.

Example. (1) Let $(G, S)$ be a spherical Coxeter system of rank $m$ and $\mathcal{A}$ the poset of all proper subsets of $S$. Then $a_{0}=\varnothing$ is the unique member of $\mathcal{A}$ of height $0, \mathcal{A}\left(>a_{0}\right)$ is an abstract $(m-2)$-polytope, and $S$ is the set of 1-cells of $\mathcal{A}$. Thus if we define $\rho: S \rightarrow G$ by $\rho(s)=s$, then $(G, \rho, \mathcal{A})$ is a thin triple of height $m-1$. The poset $P(G, \rho, \mathcal{A})$ is the Coxeter complex of the Coxeter system $(G, S)$ and it is well known that the geometric realization of the Coxeter complex of a spherical Coxeter system of rank $m$ is homeomorphic to the $(m-1)$-sphere. See for example Theorem 66.28 in [CR].

Further for $a \in \mathcal{A}$ of height $k,\left(Q_{a}, S_{a}\right)$ is well known to be a Coxeter system of rank $k$, where $S_{a}=\{s \in S: s \in a\}$, and the Coxeter complex is residually connected; cf. 29.13 in [FGT]. Thus (RM2) is also satisfied, so the Coxeter complex is a manifold triple of height $m-1$ and a sphere.

Remark. (2) We are now in a position to prove Theorem 5. Assume $(G, \rho, \mathcal{A})$ satisfies the hypotheses of Theorem 5 . Then (RM1) is satisfied by hypothesis, so it remains to verify (RM2). But this follows from Example 1.

Example. (2) Let $G$ be a group generated by a set $S$ of $m$ involutions. We construct a faithful representation of $G$ on a 2-dimensional manifold. Let $\mathcal{A}$ be the poset with least element $a_{0}$ and $\mathcal{A}\left(>a_{0}\right)$ a $2 m$-gon. Let $I$ be the set of 1-cells of $\mathcal{A}$. Pick any bijection $\rho: I \rightarrow S$. Then $(G, \rho, \mathcal{A})$ is a manifold triple of height 2. This follows from Theorem 5 . Namely as $2 m$-gons are 1 -spheres, (RM1) is satisfied. Further if $a \in \mathcal{A}$ is of height 2, then as $\mathcal{A}\left(>a_{0}\right)$ is a $2 m$ gon, $\left(a_{0}, a\right)_{\mathcal{A}}=\{b, c\}$ is of order 2 , so $\langle\rho(b), \rho(c)\rangle=Q_{a}$ is a dihedral group and $\left(Q_{a},\{\rho(b), \rho(c)\}\right)$ is a Coxeter system of rank 2. Thus (RM2) holds by Example 1.

(9.4) Let $S$ be a set of involutions generating a group $G$ such that for each $r \in S$, there exists a subset $P_{r}$ of $G$ such that

(1) $1 \in P_{r}$.

(2) $P_{r} \cap P_{r} r=\varnothing$.

(3) For $r, s \in S$ and $g \in P_{r}$, if $s g \notin P_{r}$ then $s^{g}=r$.

Then $(G, S)$ is a Coxeter system.

Proof. This is Lemma 4.2.1 in [D] and comes from page 18 of [Bb]. 
(9.5) Let $(G, \rho, \mathcal{A})$ be a thin triple, $P=P(G, \rho, \mathcal{A})$ the parabolic poset of the triple, $U=P^{1}$ the 1-skeleton of $P, I$ the set of 1-cells of $\mathcal{A}$, and $S=\rho(I)$. Then either:

(1) $(G, S)$ is a Coxeter system, or;

(2) There exists $i \in I$ such that 1 and $\rho(i)$ are in the same connected component of $U-\operatorname{Fix}_{U}(\rho(i))$.

Proof. This is essentially Lemma 4.2 in [D]; we include a proof for completeness. Assume (2) is false and for $r \in S$ define $P_{r}$ to consist of those $g \in G$ such that 1 and $g$ are in the same connected component of $Y_{r}=U-\operatorname{Fix}_{U}(r)$. We show the subsets $P_{r}$ satisfy (1), (2), and (3) of lemma 9.4, so that by that lemma, $(G, S)$ is a Coxeter system.

Recall that by definition of $P$, the 0 -cells of $P$ are the members of $G$ and $G$ acts by right multiplication on $P$, so the stabilizer of each 0-cell is trivial. In particular $G \subseteq Y_{r}$, so our definition of $P_{r}$ makes sense. For $g, h \in G$, write $\sim_{r}$ for the equivalence relation on $G$ defined by $g \sim_{r} h$ if $g$ and $h$ are in the same connected component of $Y_{r}$. Thus $P_{r}$ is the equivalence class of $\sim_{r}$ containing 1 and in particular $1 \in P_{r}$.

Next $r$ permutes the connected components of $Y_{r}$ and as (2) fails, $r \notin P_{r}$, so as $r=1 \cdot r \in P_{r} r, P_{r} r \neq P_{r}$, so $P_{r} \cap P_{r} r=\varnothing$.

Finally let $r, s \in S$ and $g \in P_{r}$ with $s g \notin P_{r}$. Then $g \sim_{r} 1 \nsim_{r} s g$, so $g \nsim_{r} s g$ and hence 1 and $s$ are in distinct connected components of $Y_{r} g^{-1}=U-$ $\operatorname{Fix}_{U}\left(r^{g^{-1}}\right)$. Now $s=\rho(i)$ for some $i \in I$ and if $Q_{i} \notin \operatorname{Fix}\left(r^{g^{-1}}\right)$ then $1<$ $Q_{i}>s$ is a path in $U-\operatorname{Fix}\left(r^{g^{-1}}\right)$, a contradiction. Thus $Q_{i} \in \operatorname{Fix}\left(r^{g^{-1}}\right)$, so as $\langle s\rangle$ is the stablizer in $G$ of $Q_{i}, r^{g^{-1}}=s$. That is $s^{g}=r$, completing the proof.

(9.6) Let $(G, \rho, \mathcal{A})$ be a 2-homology manifold triple of height $n$ with parabolic poset $X$ a 2-homology n-sphere. Let $I$ be the set of 1-cells of $\mathcal{A}$ and $S=\rho(I)$. Then $(G, S)$ is a spherical Coxeter system.

Proof. The proof comes from Proposition 4.4 in [D]. If $(G, S)$ is a Coxeter system then by $6.3, X$ is finite, so $G$ is finite and hence $(G, S)$ is spherical. Thus we may assume $(G, S)$ is not a Coxeter system, so by 9.5 there exists $i \in I$ such that 1 and $r=\rho(i)$ are in the same connected component of $U-\operatorname{Fix}_{U}(r)$, where $U=X^{1}$ is the 1-skeleton of $X$. Let $x_{1}, \ldots, x_{k}$ be a path in $U-\operatorname{Fix}_{U}(r)$ from 1 to $r$ with no repeats. Then $x_{0}, x_{1}, \ldots, x_{k}, x_{0}$ is a cycle, where $x_{0}=Q_{i}$. Hence $Z=\left\{x_{0}, \ldots, x_{k}\right\}$ is a 1 -submanifold of $X$. Notice $Z$ is lower closed since if $h\left(x_{i}\right)=1$ then $X\left(<x_{i}\right)=\left\{x_{i-1}, x_{i+1}\right\} \subset Z$. By construction the maximal members of $Z$ are 1-cells.

Next let $Y=\operatorname{Fix}_{X}(r)$. As the stabilizer in $G$ of each 0-cell is trivial, the minimal members of $Y$ are the 1-cells fixed by $r$, so $Y \cap Z=\left\{x_{0}\right\}$. As $G$ 
is upper admissible, $Y$ is upper closed. By Smith's Theorem, 6.4, $Y$ is a 2-homology $(n-1)$-manifold. By 6.6, $Y$ and $Z$ are orientable. But now 9.1 says $H_{1}\left(X, \mathbf{F}_{2}\right) \neq 0$, contradicting $X$ a 2-homology $n$-sphere.

Remark. (3) Notice 9.6 implies Theorem 3.

(9.7) If $(G, \rho, \mathcal{A})$ is a 2-homology manifold triple and $a \in \mathcal{A}$, then $\left(Q_{a}, \rho(I(a))\right)$ is a spherical Coxeter system.

Proof. Observe $\left(Q_{a}, \rho_{a}, \mathcal{A}(\leq a)\right)$ is a 2-homology manifold triple whose parabolic poset is a 2-homology sphere, so by $9.6,\left(Q_{a}, \rho(I(a))\right)$ is a spherical Coxeter system.

\section{Regular 2-homology manifolds which are spheres.}

In this section $X$ is a poset of finite height $n$ and $G$ is an upper admissible group of automorphisms of $X$ transitive on 0-cells. Assume $X$ is a 2-homology $n$-manifold and a 2-homology $n$-sphere. Let $a_{0}$ be a 0 -cell of $X$, $\mathcal{A}=X\left(\geq a_{0}\right), I$ the set of 1 -cells in $\mathcal{A}, \rho(i)$ the reflection through $i \in I$, and $R=\{\rho(i): i \in I\}$. By 9.3, $(G, \rho, \mathcal{A})$ is a 2-homology manifold triple and $X$ is isomorphic to the parabolic poset of $(G, \rho, \mathcal{A})$.

Let $\operatorname{Pow}(I)$ be the power set of $I$ and $\phi: \mathcal{A} \rightarrow \operatorname{Pow}(I)$ be the map $\phi(a)=I(a)$ for $a \in \mathcal{A}$.

Let $G_{a}$ be the stabilizer in $G$ of $a \in \mathcal{A}$. By 4.4, $G_{a}=P_{I(a)}$ is the parabolic generated by $R_{a}=\rho(I(a))$, the set of reflections through the set $I(a)$ of 1-cells between $a_{0}$ and $a$. Throughout this section we assume:

Hypothesis 10.1. Distinct 0 -cells of $X$ are below at most one 1-cell of $X$.

(10.2) $\rho: I \rightarrow R$ is a bijection.

Proof. If $\rho(i)=\rho(j)$ then $a_{0}, a_{0} \rho(i) \leq i, j$, so as distinct 0 -cells are below at most one 1 - cell, $i=j$. Indeed the injectivity of $\rho$ is equivalent to Hypothesis 10.1 .

(10.3) $(G, R)$ is a spherical Coxeter system.

Proof. This is 9.6.

Let $x \in \mathcal{A}$ be of height $m<n, L=I(x)$,

$$
M=\bigcup_{y>x} I(y)-L
$$


$H=P_{M}$ the parabolic of $G$ determined by $M$, and $Y$ the subset of $X$ which is the image of $\mathcal{B}=\mathcal{A}(\geq x)$ under $H$. Assume

$$
|I(y)|=|I(x)|+1 \text { for each }(m+1)-\text { cell y above } x .
$$

Then $Y$ is upper closed, the minimal members of $Y$ are $m$-cells, and $Y$ is an abstract polytope.

Proof. As $\mathcal{B}$ is upper closed, so is $Y$ and by construction the minimal members of $Y$ are the $m$-cells $x H$ of $X$.

Next $H$ is an upper admissible group of automorphisms of the poset $Y$ transitive on the set $x H$ of 0 -cells of $Y$ with $\mathcal{B}=Y(\geq x)$, so setting $\mathcal{H}=$ $\left(H_{b}: b \in \mathcal{B}\right), 3.2$ says $Y$ is isomorphic to $P(\mathcal{H})$ and $\mathcal{H}$ is a compatible family of subgroups of $H$. We verify the hypotheses of 4.3 for $H, \mathcal{H}$, and conclude from that lemma that $Y \cong P(\mathcal{H})$ is an abstract polytope. First $\mathcal{B}(>x)$ is a 2-homology $(n-m-1)$-manifold and sphere, and hence an abstract polytope as $\mathcal{A}\left(>a_{0}\right)$ is a 2-homology $(n-1)$-manifold and sphere. Second $H_{x}=P_{M} \cap P_{L}=P_{M \cap L}$ as $(G, R)$ is a Coxeter system. But $M \cap L=\varnothing$, so $P_{L \cap M}=1$.

Similarly for $a \in \mathcal{B}$ of $\mathcal{B}$-height $1, G_{a}=P_{I(a)}$ with $I(a)=L \cup\{i(a)\}$ for some $i(a) \in M$ by hypothesis $(*)$. Now

$$
H_{a}=P_{M} \cap P_{I(a)}=P_{M \cap I(a)}=P_{i(a)}=\langle\rho(i(a))\rangle
$$

so $H_{a}$ is of order 2. Similarly for $b \in \mathcal{B}$,

$$
\begin{aligned}
H_{b}=P_{M} \cap P_{I(b)}=P_{M \cap I(b)}=\left\langle P_{i(a)}: a \leq b\right. & \text { and } \left.h_{\mathcal{B}}(a)=1\right\rangle \\
& =\left\langle H_{a}: a \leq b \text { and } h_{\mathcal{B}}(a)=1\right\rangle .
\end{aligned}
$$

Finally

$$
H=P_{M}=\left\langle P_{i(a)}: h_{\mathcal{B}}(a)=1\right\rangle=\langle\mathcal{H}\rangle .
$$

Thus we have shown that $Y$ is an abstract polytope, completing the proof.

Define a $k$-subset $J$ of $I$ to be $\mathcal{A}$-simplicial if

$$
\phi: \phi^{-1}(\operatorname{Pow}(J)) \cap \mathcal{A}^{k} \rightarrow \operatorname{Pow}(J)
$$

is an isomorphism. Define $\lambda: \operatorname{Pow}(I) \rightarrow \mathbf{Z}$ by

$$
\lambda(J)=\max \left\{m \leq k: L \text { is } \mathcal{A} \text {-simplicial for all } L \in \operatorname{Pow}(J)^{m}\right\} .
$$


(10.5) Let $J$ be an $(m+1)$-subset of $I$ with $\lambda(J) \geq m-1$ and assume each $m$-subset $L$ of $J$ is of the form $I\left(c_{L}\right)$ for some $m$-cell $c_{L}$ with $\phi: \mathcal{A}\left(\leq c_{L}\right) \rightarrow$ Pow $(L)$ an isomorphism. Let

$$
\mathcal{D}=\phi^{-1}\left(\operatorname{Pow}(J)^{m-1}\right) \cup\left\{c_{L}: L \text { is an m-subset of } J\right\},
$$

$D=P_{J}$, and $Z$ the subset of $X$ consisting of all D-conjugates of members of $\mathcal{D}$. Then $Z$ is a lower closed subset of $X^{m}$ which is a 2-homology $m$ manifold.

Proof. The proof is much the same as that of the previous lemma. As $\mathcal{D}$ is lower closed with $\mathcal{D} \subseteq X^{m}$, so is $Z$. Our hypotheses say $\phi: \mathcal{D} \rightarrow \operatorname{Pow}(J)^{m}$ is an isomorphism. In particular $\mathcal{D}\left(>a_{0}\right)$ is a 2-homology $(m-1)$-sphere and for $c \in \mathcal{D}, G_{c}=P_{I(c)}=D_{c}$. Now complete the proof as in the previous lemma.

(10.6) Let $0 \leq h \leq n$ and assume:

(*) For all $x \in \mathcal{A}^{h}$ and $y \in X$ with $x<y$ that $I(x) \neq I(y)$.

Then either

(1) $\phi: \mathcal{A}^{h} \rightarrow \operatorname{Pow}(I)^{h}$ is an isomorphism. Further if $h<n$ then $\phi:$ $\mathcal{A}^{h+1} \rightarrow \operatorname{Pow}(I)^{h+1}$ is a surjection, while if $h=n$ then $|I|=n+1$ and $X$ is isomorphic to the Coxeter complex for $(G, R)$, or

(2) $n=h, X$ has exactly two $n$-cells $u$ and $v,|I|=n, X=\{u, v\} * X^{n-1}$, $\phi: \mathcal{A}^{n-1} \rightarrow \operatorname{Pow}(I)^{n-1}$ is an isomorphism, and $X^{n-1}$ is isomorphic to the Coxeter complex for $(G, R)$.

Proof. We prove the lemma by induction on $h$ and $n$. For $h=0$, (1) holds as $a_{0}$ is the unique 0 -cell of $\mathcal{A}$ and the empty set is the unique 0 -subset of $I$. So a minimal counter example satisfies $n \geq h>0$.

If $\phi$ is an isomorphism of $\mathcal{A}^{h}$ with $\operatorname{Pow}(I)^{h}$ and $h \neq n$, let $k=h+1$, while if $\phi$ is not an isomorphism, let $k=h$. If $\phi$ is an isomorphism and $h=n$, then as $\mathcal{A}\left(>a_{0}\right)$ is a 2-homology $(n-1)$-sphere, $|I|=n+1$. But now $X$ is isomorphic to the Coxeter complex for $(G, R)$ and (1), holds, so if $h=n$ we may assume $\phi$ is not an isomorphism.

Let $J$ be a $k$-subset of $I$ and $m=k-1$. If $m=h$ then by hypothesis, $\phi$ : $\mathcal{A}^{m} \rightarrow \operatorname{Pow}(I)^{m}$ is an isomorphism, while if $m<h$ this holds by minimality of $h$.

Suppose first $J \neq I(a)$ for some $k$-cell $a$ of $\mathcal{A}$. Define $\mathcal{D}, D$, and $Z$ as in 10.5. By the previous paragraph, the map $c \mapsto I(c)$ is an isomorphism between $\mathcal{D}^{m}$ and $\operatorname{Pow}(J)^{m}$, so by $10.5, Z$ is a lower closed 2-homology $m$ manifold with maximal elements the images $c D$ under $D$ of the $m$-cells $c$ in $\mathcal{D}$. 
Notice that if $y$ is an $k$-cell then $\left(G_{y}, \rho_{y}, \mathcal{A}(<y)\right)$ satisfies our hypotheses and is of height $m<n$, so by minimality of $n, \mathcal{A}(<y) \cong \operatorname{Pow}(I(y))^{m}$ and $I(y)$ is of order $k$.

Pick $j_{0} \in J$ and let $L=J-\left\{j_{0}\right\}$. We have seen there is an $m$-cell $x$ with $I(x)=L$. By the previous paragraph, if $y$ is an $k$-cell then $|I(y)|=k=$ $m+1=|I(x)|+1$. Thus we may apply the construction of 10.4 to this $x$ and obtain a subgroup $H$ of $G$ and a thin residually closed subcomplex $Y$ which is upper closed with minimal elements $x H$. By $6.6, Z$ and $Y$ are orientable.

Now $x \in Y \cap Z$ and as $Z \subseteq X^{m}$ and the minimal members of $Y$ are in $x H$, if $y \in Y \cap Z$ then $y=x g$ for some $g \in H$. But also $y=c d$ for some $d \in D$ and some $m$-cell $c$ of $Z$, so $c=x$ and $g \in G_{x} d=P_{L} d \subseteq D=P_{J}$, as $L \subset J$. Thus

$$
g \in H \cap D=P_{M} \cap P_{J}=P_{M \cap J} .
$$

But $J=L \cup\left\{j_{0}\right\}$ with $L \cap M=\varnothing$ and $j_{0} \notin M$ as $J \neq I(u)$ for any $u$. Thus $g \in H \cap D=1$, so $y=x$. That is $Y \cap Z=\{x\}$. But now 9.1 supplies a contradiction.

Thus we have established the surjectivity of our map. In particular if $k=h+1$ then (1) holds, so from now on we may take $k=h$.

Next suppose $I(u)=J=I(v)$ for distinct $h$-cells $u$ and $v$. We saw above that $\phi: \mathcal{A}(\leq w) \rightarrow \operatorname{Pow}(J)^{m}$ is an isomorphism for $w=u, v$, so $X(<u)=X(<v)=Z$. Thus $Z_{0}=\{u, v\} \cup Z=\{u, v\} * Z$ is a lower closed 2-homology $h$ - manifold. Now if $h=n$ then as $X(>x)$ is of order 2 for each $m$-cell $x \in Z, X(>x)=\{u, v\}$. But now if we let $\Gamma$ consist of $n$ and $n-1$ cells of $X$ and $\Sigma=\Gamma \cap Z_{0}$, then $\Gamma(y) \subseteq \Sigma$ for each $y \in \Sigma$, so as $\Gamma$ is connected, $\Gamma=\Sigma$ and hence $X=Z_{0}$, so that (2) holds. Thus we may assume $h<n$.

Let $y$ be an $(h+1)$-cell. Then $\left(G_{y}, \rho_{y}, \mathcal{A}(<y)\right)$ satisfies our hypotheses and is of height $h$, so by a previous case either $I(y)$ is of order $h+1$ and

$$
\phi: \mathcal{A}(<y) \rightarrow \operatorname{Pow}(I(y))^{h}
$$

is an isomorphism, or $\mathcal{A}(<y)$ has just $2 h$ - cells $a$ and $b$ and $I(y)=I(a)=$ $I(b)$. But the latter case contradicts hypothesis $(*)$, so the former holds.

Given this observation, we can repeat an argument above on $u$ in place of $x$ to obtain a thin residually connected upper closed subcomplex $Y_{0}$ with $Y_{0} \cap Z_{0}=\{u\}$. Hence once again 9.1 supplies a contradiction, completing the proof.

Remark. Notice that Theorem 10.6 implies Theorem 4. 


\section{Hirzebruch's Prize Question.}

In [FLM], Frenkel, Lepowsky, and Meurman construct a vertex operator algebra whose automorphism group is the largest sporadic group the Monster. Vertex operator algebras are the algebraic version of physicist's conformal field theory. There are also geometric versions of conformal field theory, although as I understand it, no geometric formalism yet exists for conformal field theory which is satisfactory to mathematicians. However some kind of metaprinciple says there should exist a geometric object corresponding to the Monster vertex operator algebra.

In that direction Hirzebruch has posed the following problem in [HBJ]:

Prize Question: Does there exist a 24-dimensional compact, orientable, differentiable manifold $X$ (admitting the action of the Monster) with $p_{1}(X)=$ $0, w_{2}(X)=0, \hat{A}(X)=1$, and $\hat{A}\left(X, T_{\mathbf{C}}\right)=0$ ?

Here $p_{1}(X)$ is the first Pontrjagin class of $X, w_{2}(X)$ is the second StiefelWhitney class, $\hat{A}(X)$ is the $\hat{A}$-genus of $X$, and $\hat{A}\left(X, T_{\mathbf{C}}\right)$ is the twisted $\hat{A}$-genus of the complex tangent bundle $T_{\mathbf{C}}=T X \otimes \mathbf{C}$.

Theorem 5 applies to groups $G$ which are close to being spherical Coxeter groups, in the sense that $G$ is generated by a set $S$ of involutions such that for many subsets $J$ of $S,(\langle J\rangle, J)$ is a spherical Coxeter system. Examples of such groups include certain subgroups of the Bimonster, including the Monster. Thus Theorem 5 can be used to construct representations of the Monster on manifolds.

The Bimonster is the wreath product of the Monster by a group of order 2. Thus the Bimonster $B$ has a normal subgroup $M_{1} \times M_{2}$ of index 2 and $B$ is $M_{1} M_{2}$ extended by an involution $t$ interchanging $M_{1}$ and $M_{2}$. In particular $B$ is transitive on the involutions in $B-M_{1} M_{2}$ and $C_{M_{1} M_{2}}(t)$ is a diagonal subgroup of $M_{1} \times M_{2}$ isomorphic to the Monster.

In [CNS], Conway, Norton, and Soicher show that there exists a set $\Gamma \subseteq$ $B-M_{1} M_{2}$ of 26 involutions generating $B$ and admitting the structure of a projective plane of order 3 in such a way that if $i, j \in \Gamma$ are distinct then $|i j|=3$ if $i$ and $j$ are incident in $\Gamma$ and $|i j|=2$ otherwise. Further the full group $A$ of automorphisms of the projective plane is induced on $\Gamma$ in $B$. Call $\Gamma$ a set of Conway-Norton-Soicher involutions.

We have the usual Coxeter diagram $\Delta$ defined on $\Gamma$. Define a subset $J$ of $\Gamma$ to be spherical if the restriction $\Delta_{J}$ of $\Delta$ to $J$ is spherical and define $J$ to be $B$ - spherical if $(\langle J\rangle, J)$ is a spherical Coxeter system. Thus if $J$ is $B$-spherical then $J$ is spherical. We seek manifold triples $(G, \rho, \mathcal{A})$ with $G \leq B$ and $\rho(I) \subseteq \Gamma$ satisfying the hypotheses of Theorem 5 . Thus if $a \in \mathcal{A}$ then $\mathcal{A}(\leq a)$ is simplicial and $\rho(I(a))$ is a $B$-spherical subset of $\Gamma$.

In Section 12, we record various results about the action of $A$ on spherical 
and $B$-spherical subsets of $\Gamma$. Then in Section 13 we use Theorem 5 and these results to construct manifolds admitting the action of subgroups $G$ of the Bimonster with dimensions ranging from 5 to 14 . Notice that the height of our triple $(G, \rho, \mathcal{A})$ is $|I(a)|$ for $a$ a maximal cell of $\mathcal{A}$ and hence bounded by 16 by Lemma 12.1 . The actual bound when $G$ is the Monster or Bimonster is smaller; 14 may be best possible. Namely by 12.1, the only class of spherical sets of rank 16 has diagram $4 D_{4}$. Up to conjugation it consists of all points distinct from a fixed point $p$ plus the four lines through $p$. But it is not $B$-spherical by 12.3 , so the bound of 16 cannot be attained.

The geometric realization $T(L)$ of any link $L$ in any poset $P=P(G, \rho, \mathcal{A})$ we construct is smoothly isomorphic to a sphere, so $T(P)$ is a topological manifold admitting a canonical smooth differential structure. As $G$ has a subgroup $G_{*}$ of index 2 with $\rho(I) \subseteq G-G_{*}, T(P)$ is orientable. When $G=B$, the centralizer in $G$ of each $t \in \Gamma$ is a copy of the Monster, and Smith Theory says the fixed points $T$ of $t$ on $T(P)$ is a 2- homology manifold of dimension $h(P)-1$, which then admits the action of $C_{M_{1} M_{2}}(t)$, a group isomorphic to the Monster. Presumably this space is actually a manifold. Then for example in the case when $T(P)$ is 9-dimensional, we could take the product of three copies of $T$ to obtain a 24-dimensional manifold admitting the Monster.

However the manifolds we construct are unfortunately not candidates for the Hirzebruch manifold. This is because our reflections are inducing orientation reversing diffeomorphisms of these manifolds, so the Pontrjagin numbers of the manifolds are 0 , and hence the $\hat{A}$-genus is 0 rather than 1 .

\section{The poset of spherical subsets of the plane of order 3 .}

Let $V$ be a 3-dimensional vector space over the field $F$ of order 3 and $\pi$ the projective plane over the field of order 3 . Thus $\pi$ consists of the points (1-dimensional subspaces of $V$ ) and lines (2-dimensional subspaces of $V$ ) of $V$ together with the incidence relation of inclusion between points and lines.

Associate a Coxeter matrix $M=\left(m_{i, j}\right)$ of size 26 to $\pi$ by letting $m_{i, i}=1$ and for $i \neq j$ let $m_{i, j}=3$ if $i$ is incident to $j$ in $\pi$ and $m_{i, j}=2$ otherwise. Then each subset $J$ of $\pi$ inherits a Coxeter diagram $\Delta_{J}$ from the Coxeter diagram defined on $\pi$ by the Coxeter matrix $M$. A subset $J$ is spherical if its Coxeter diagram is spherical. The type of $J$ is the type of its Coxeter diagram $\Delta_{J}$. Write $J^{\perp}$ for the set of vertices $i \in \pi$ orthogonal to each member of $J$; that is $m_{i, j}=2$ for each $j \in J$.

Let $P$ be the poset of all spherical subsets of $\pi$ partially ordered by inclusion. We call the members of $P$ of order $k, k$-cells. We regard the empty set as spherical, so $\phi$ is the unique 0 -cell. The $k$-cells are the members of $P$ of 
height $k$.

Let $A$ be the group of all automorphisms of $\pi$. Thus $A=L\langle\tau\rangle$ where $L=P S L(V) \cong L_{3}(3)$ is the projective special linear group on $V$ and $\tau$ is a polarity of $\pi$. Thus $\tau$ induces an outer automorphism on $L$ and $A=$ $\operatorname{Aut}(L) \cong \operatorname{Aut}\left(L_{3}(3)\right)$.

We record the following two results without proof. They can be retrieved from tables in Section 11 of [CNS], although those tables are supplied without proof too. Alternatively, proofs of 12.1 and 12.2 appear in $[\mathbf{A}]$.

(12.1) The maximal cells of $P$ have the following types and $A$ is transitive on cells of each type:

(1) $13 A_{1}$ of rank 13 .

(2) $4 D_{4}$ of rank 16 .

(3) $3 A_{5}$ of rank 15.

(4) $A_{11} \oplus A_{4}$ of rank 15 .

(5) $D_{5} \oplus 3 A_{3}$ of rank 14 .

(6) $D_{6} \oplus 2 A_{3} \oplus 2 A_{1}$ of rank 14 .

(7) $D_{8} \oplus 2 A_{3}$ or rank 14 .

(8) $\quad E_{6} \oplus 2 A_{2} \oplus A_{3}$ of rank 13 .

(9) $E_{7} \oplus A_{1} \oplus A_{2} \oplus A_{3}$ of rank 13 .

(10) $E_{8} \oplus A_{2} \oplus A_{3}$ of rank 13 .

(12.2) A is transitive on connected spherical cells of each type.

(12.3) A spherical subset $J$ of $I$ is not $B$-spherical if and only if $Y \subseteq J \subseteq$ $X$, where $X$ is maximal spherical and $Y$ is one of the following sums of connected components of $X: 2 D_{4}, D_{6} \oplus 2 A_{1}, E_{7} \oplus A_{1}, D_{8}$, or $E_{8}$. Further if $\sigma: W \rightarrow\langle\rho(Y)\rangle$ is the corresponding Coxeter cover then $\operatorname{ker}(\sigma)=\left\langle z_{1} \cdots z_{r}\right\rangle$ where $z_{i}$ generates the center of the ith direct factor of $W$.

Proof. A stronger assertion seems to be made on page 44 of [CNS], but the assertion given here is the best that could be possible and is presumably what the authors of [CNS] intended.

Let $J$ be spherical but not $B$-spherical. Then $J \subseteq X$ maximal spherical. Then $J \subseteq X_{1} \cup \cdots \cup X_{r}$ with $X_{i}$ a connected component of $X$ and $J_{i}=$ $X_{i} \cap J \neq \varnothing$. Let $H_{i}=\left\langle\rho\left(J_{i}\right)\right\rangle$ and $\sigma: W \rightarrow H=H_{1} \cdots H_{r}$ the Coxeter covering of $H$ and $W=W_{1} \times \cdots W_{r}, \sigma\left(W_{i}\right)=H_{i}$.

From 12.2, $A$ is transitive on connected spherical subsets $Z$ of $I$ of each type and from [CNS], particularly Table $3, Z$ is $B$-spherical except when $Z$ is of type $D_{8}$ or $E_{8}$, where the kernel of the Coxeter covering is the center of order 2. For example each $Z$ is a subset of a maximal connected subset of type $A_{11}, D_{8}$ or $E_{8}$ and all subsets of $A_{11}$ and all proper subsets of $D_{8}$ or $E_{8}$ 
are $B$-spherical. Thus unless $X_{i}=J_{i}$ is of type $D_{8}$ or $E_{8}, J_{i}$ is $B$-spherical, and we may assume the latter.

Then $\left[\operatorname{ker}(\sigma), W_{i}\right] \leq \operatorname{ker}(\sigma) \cap W_{i}=1$, so $\operatorname{ker}(\sigma) \leq Z(W)$. Hence the projection $z_{i}$ of $z \in \operatorname{ker}(\sigma)$ on $W_{i}$ is contained in $Z\left(W_{i}\right)$ of order at most 2 . Further if $z_{i} \neq 1$ then $\sigma\left(z_{i}\right)=\sigma\left(z z_{i}^{-1}\right) \in C_{H}\left(\left\langle\rho\left(X_{i}\right)\right\rangle\right)$ so $J_{i}=X_{i}$. Hence if $J$ is chosen to be minimal non $B$-spherical then $\operatorname{ker}(\sigma)=z_{1} \ldots z_{r}$ with $z_{i}$ of order 2 generating $Z\left(W_{i}\right)$ and $J_{i}=X_{i}$. It follows that $J_{i}$ is of type $A_{1}, D_{4}$, $D_{6}, D_{8}, E_{7}$, or $E_{8}$. If $J_{i}$ is of type $D_{8}$ or $E_{8}$ then $J=J_{i}$ by minimality of $J$ and the lemma holds. If $J_{i}$ of type $D_{4}$ then all components of $X$ are of type $D_{4}$ and from page 44 of [CNS], $2 D_{4}$ is not $B$-spherical, so the lemma holds. If $J_{1}$ is of type $D_{6}$ then $J_{1}^{\perp}=3 A_{3} \oplus 2 A_{1}$, so as $Z\left(W_{i}\right) \neq 1, J_{i}$ must be of type $A_{1}$ for $i>1$ and then from page 44 of [CNS], $J$ is minimal non $B$-spherical if and only if $J$ is of type $D_{6} \oplus 2 A_{1}$. A similar argument works for $J_{1}$ of type $E_{7}$. This leaves the case $J$ of type $k A_{1}$. From 12.1 and the cases eliminated, $X$ is of type $13 A_{1}$, which is $B$ - spherical from the discussion at the top of page 45 of $[\mathrm{CNS}]$.

\section{Manifolds for subgroups of the Bimonster.}

Continue the hypotheses and notation of Section 12. In particular $V$ is a 3dimensional vector space over the field $F$ of order $3, \pi$ is the projective plane of $V$, and $P$ is the poset of all spherical subsets of $\pi$ ordered by inclusion.

We construct manifold triples $(G, \rho, \mathcal{A})$ with $G$ a subgroup of the Bimonster $B$, and $\mathcal{A}$ a subposet of $P$, and $I \subseteq \pi$. The map $\rho: I \rightarrow B$ is induced by the Conway-Norton-Soicher embedding. As $\mathcal{A}$ is a subposet of $P$, the map $\phi: \mathcal{A} \rightarrow \operatorname{Pow}(I)$ is an injection whose image is contained in $P$ and for $a \in \mathcal{A}, \mathcal{A}(\leq a) \cong \operatorname{Pow}(I(a))$ via $\phi$. We choose $\mathcal{A}$ so that its members are $B$-spherical and hence $P\left(G_{a}, \rho_{a}, \mathcal{A}(\leq a)\right)$ is the Coxeter complex of the sphericial Coxeter system $\left(G_{a}, \rho(I(a))\right)$, and thus is a sphere.

Remark 1. Let $I \subseteq \pi, G=\langle\rho(I)\rangle, I=I_{1} \cup \cdots \cup I_{m}$ a partition of $I$ with $\left|I_{i}\right|>1$ for each $i$, and $K_{i}$ a simplicial complex with vertex set $I_{i}$ such that $K_{i}$ is a homology sphere and manifold. Let $\mathcal{B}_{i}=s d\left(K_{i}\right)$ be the barycentric subdivision of $K_{i}$ regarded as a poset, and let $\mathcal{A}_{i}=\mathcal{B}_{i} \cup\{\varnothing\}$ be the extended poset with unique 0 -cell $\varnothing$ and $\mathcal{B}_{i}=\mathcal{A}_{i}(>\varnothing)$. Finally let $\mathcal{A}=\mathcal{A}_{1} \times \cdots \times \mathcal{A}_{m}$ be the product poset consisting of all subsets $J=J_{1} \cup \cdots \cup J_{m}$ with $J_{i} \in \mathcal{A}_{i}$. Then

$$
h(\mathcal{A})=\sum_{i=1}^{m} h\left(\mathcal{A}_{i}\right)
$$

and $\mathcal{A}(>\varnothing)$ is the join $\mathcal{B}_{1} \cup \cdots \cup \mathcal{B}_{m}$ of homology spheres and manifolds, so $\mathcal{A}(>\varnothing)$ is a homology sphere and manifold. Thus if each cell $J$ in $\mathcal{A}$ is 
$B$-spherical, then by remarks above and Theorem $5,(G, \rho, \mathcal{A})$ is a manifold triple of height $h(\mathcal{A})$.

Notice that to check that $J$ is $B$-spherical amounts by 12.3 to checking that the diagram of $J$ is spherical and contains none of the excluded subdiagrams $2 D_{4}, D_{6} \oplus 2 A_{1}, E_{7} \oplus A_{1}, D_{8}$, or $E_{8}$. In particular if $\Delta_{J}$ is spherical and contains no $2 D_{4}$ or $D_{6}$ subdiagrams then $J$ is $B$-sphericial.

Remark 2. In all but the last example in this section, $\mathcal{A}_{i}$ is the poset of all proper subsets of $I_{i}$ and hence $\mathcal{A}_{i}(>\varnothing)$ is a sphere and manifold and $h\left(\mathcal{A}_{i}\right)=\left|I_{i}\right|-1$. Hence

$$
h(\mathcal{A})=\sum_{i=1}^{m} h\left(\mathcal{A}_{i}\right)=\sum_{i=1}^{m}\left(\left|I_{i}\right|-1\right)=|I|-m .
$$

In each case if $J \in \mathcal{A}$ then $\Delta_{J}$ contains no $2 D_{4}$ or $D_{6}$ subdiagram, so by Remark $1, J$ is $B$-spherical and $(G, \rho, \mathcal{A})$ is a manifold triple of height $|I|-m$.

Example 1. Let $I$ have the $Y_{555}$ diagram of [CNS]. That is $|I|=16$ and the diagram of $I$ is a "Y" with a central node and three legs with $A_{5}$ diagrams connected to the central node at one end. From [CNS], $\langle\rho(I)\rangle=B$ is the Bimonster. Let $I_{1}$ be any subset of $I$ of order at least 2 containing the central node and contained in the unique subset of $I$ whose diagram is $D_{4}$. Thus the diagram of $I_{1}$ is $A_{2}, A_{3}$, or $D_{4}$. Finally let $I=I_{1} \cup \cdots \cup I_{m}$ be a partition of $I$ containing $I_{1}$ with $\left|I_{i}\right|>1$ for each $i$.

Then the diagram of each $J \in \mathcal{A}$ is the sum of diagrams of type $A$, so it is spherical and contains no subdiagram of type $2 D_{4}$ or $D_{6}$. Hence by Remark 1 , we have a manifold triple $(G, \rho, \mathcal{A})$ of height $|I|-m=16-m$. As $m$ can range between 2 and 8 , we get manifolds of dimension between 8 and 14 by varying our partition.

Let $X=P(B, \rho, \mathcal{A})$. For $t \in \rho(I), M=C_{M_{1} M_{2}}(t)$ is the Monster and acts faithfully on the 2-homology manifold $\operatorname{Fix}_{X}(t)$ of dimension $h(X)-1$, ranging between 7 and 13 .

Example 2. Let $I$ have the $Y_{553}$ diagram. From [CNS]

$$
G=\langle\rho(I)\rangle=C_{B}(t) \cong \mathbf{Z}_{2} \times M
$$

where $M$ is the Monster and $t \in \Gamma$. We proceed as in Example 1, obtaining partitions of $I$ into $m$ parts, $2 \leq m \leq 7$, and manifolds of dimension $|I|-m=$ $14-m$ between 7 and 12 .

Example 3. Let $I$ have the $Y_{443}, Y_{432}$, or $Y_{332}$ diagram. Then from [CNS], $G=\langle\rho(I)\rangle$ is isomorphic to $M(24) / \mathbf{Z}_{2}, M(23) \times \mathbf{Z}_{2}$, or $M(22) / E_{4}$, respectively, so $G$ is essentially a Fischer group. Proceeding as in Examples 1 and 
2, we obtain manifolds for these groups with dimensions ranging between 5 and 10 .

Example 4. This example is a little different. Choose $I$ to have the diagram in Figure 1.b on page 28 of [CNS]. Then $G=\langle\rho(I)\rangle$ is again isomorphic to $\mathbf{Z}_{2} \times M$. There is a subset $I_{1}$ of $I$ of order 6 with diagram of type $\tilde{A}_{5}$ and $I$ is obtained by adding three legs with diagrams of type $A_{3}$. Consider any partition $I=I_{1} \cup \cdots \cup I_{m}$ containing $I_{1}$ with $\left|I_{i}\right|>1$. For $i \neq 1$ let $\mathcal{A}_{i}$ be the poset of all proper subsets of $I_{i}$, but form $\mathcal{A}_{1}$ as in Remark 1 with respect to the 1-dimensional complex $K_{1}$ whose 1 -simplices are the edges in the $\tilde{A}_{5}$ diagram on $I_{1}$. Thus $K_{1}$ is a 1 -sphere, so by Remark 1 we get a manifold triple $(G, \rho, \mathcal{A})$ of height

$$
2+|I|-6-(m-1)=12-m
$$

producing manifolds for $\mathbf{Z}_{2} \times M$ of dimension between 7 and 10 .

The preprint $[\mathbf{A}]$ contains other examples.

\section{References}

[A] M. Aschbacher, Homology manifolds with cell structure, preprint.

[CCC] - Combinatorial cell complexes, Advanced Studies in Pure Math., 24 (1996), $1-80$.

[FGT] _ Finite group theory, Cambridge U. Press, Cambridge, 1986.

[Bb] N. Bourbaki, Groupes et algèbres de Lie, Chapitres 4,5 et 6, Hermann, Paris, 1986.

[Bo] A. Borel, Seminar on transformation groups, Princeton U. Press, Princeton, 1960.

[Br] G. Bredon, Introduction to compact transformation groups, Academic Press, New York, 1972.

[CNS] J. Conway, S. Norton and L. Soicher, The Bimonster, the group $Y_{555}$, and the projective plane of order 3, Computers in Algebra, (ed. M. Tangora), Marcel Dekker, (1988), 27-50.

[CR] C. Curtis and I. Reiner, Methods of representation theory, Vol. 2, Wiley, New York, 1987.

[D] M. Davis, Regular convex cell complexes, Geometry and Topology, (ed. C. McCrory and T. Shifrin), Marcel Dekker, New York, (1987), 53-83.

[FLM] I. Frenkel, J. Lepowsky and A. Meurman, Vertex operator algebras and the Monster, Academic Press, San Diego, 1988.

[HBJ] F. Hirzebruch, T. Berger and R. Jung, Manifolds and modular forms, Max-PlankInstitute für Mathematik, Bonn, 1992.

[M] J. Munkres, Elements of algebraic topology, Addison-Wesley, Reading, 1984. 
[MS] P. McMullen and E. Schulte, Constructions for regular polytopes, J. Comb. Th. Ser. A, 53 (1990), 1-28.

This work was partially supported by NSF DMS-9101237 and NSF DMS-9622843.

California Institute of Technology

Pasadena, CAlifornia 91125

E-mail address: asch@cco.caltech.edu 